\title{
1 Audiovisual detection at different intensities 2 and delays
}

Chandramouli Chandrasekaran

4 Neuroscience Institute \& Department of Psychology, Princeton University, USA

5 Department of Electrical Engineering, Stanford University, USA

Matthias Gondan

7 Department of Psychology, University of Copenhagen, Denmark

8 Faculty for Psychology, University of Vienna, Austria

\section{Correspondence address}

10 Matthias Gondan

11 Faculty for Psychology

12 University of Vienna

13 Liebiggasse 5

141010 Vienna

15 Austria

16 Email: matthias.gondan-rochon@univie.ac.at

\section{Running Title}

18 Audiovisual detection 


\section{Abstract}

20 In divided-attention tasks with two classes of target stimuli (e.g., auditory and visual), redundancy

21 gains are typically observed if both targets are presented simultaneously, as compared with single-

22 target presentation. Different models explain such redundant-signals effects, including race and

23 coactivation models. Here we generalize one such coactivation model, the superposition model, and

24 show that restricting this evidence accumulation process to a limited time interval, that is a temporal

25 deadline, provides the model the ability to describe the detection behavior of two monkeys across a

26 wide range of intensities and stimulus onset asynchronies. We present closed-form solutions for the

27 mean absorption times and probabilities for the two-stage diffusion process with drift towards a

28 single barrier in the presence of a temporal deadline.

\section{Keywords}

30 Multisensory processing; Monkey; Decision making; Diffusion processes; Reaction times. 


\section{Background}

32 Integrating information from different senses improves perception and action, speeds up response

33 time (RT), enhances detection and discrimination accuracy, and facilitates correct arm movements

34 (Cluff, Crevecoeur, \& Scott, 2015; Crevecoeur, Munoz, \& Scott, 2016; Diederich \& Colonius, 2004a;

Miller, 1982; Nickerson, 1973; Sakata, Yamamori, \& Sakurai, 2004; Schwarz, 1989, 1994; Seilheimer,

36 Rosenberg, \& Angelaki, 2014; Stein \& Stanford, 2008; van Atteveldt, Murray, Thut, \& Schroeder,

2014). Despite many studies of multisensory integration, several questions about how the behavioral benefits of multisensory integration emerge remain unanswered (Chandrasekaran, 2016; Cluff et al., 2015; Crevecoeur et al., 2016).

In this study, we focused on the question of how the behavioral benefits of multisensory

integration vary as a function of both the sensory intensities as well as delays between the stimuli.

42 With few exceptions (Gondan, Götze, \& Greenlee, 2010), past studies have largely investigated these

43 two aspects in isolation (Cluff et al., 2015; Crevecoeur et al., 2016; Diederich \& Colonius, 2004a; Dixon

44 \& Spitz, 1980; Holmes, 2009; Meredith, Nemitz, \& Stein, 1987; Stein, Meredith, Huneycutt, \&

McDade, 1989; van Wassenhove, Grant, \& Poeppel, 2007). In this study, we extended our recent

46 paradigm where monkeys detect visual, auditory, and audiovisual vocalizations in a background of

47 noise (Chandrasekaran, Lemus, \& Ghazanfar, 2013; Chandrasekaran, Lemus, Trubanova, Gondan, \&

48 Ghazanfar, 2011; Miller, 1986) by incorporating delays between the sensory stimuli (Miller, 1986). We

49 measured the response times (RTs) and detection accuracy of the monkeys and then modeled the

50 behavioral patterns by expanding on the coactivation modeling framework (Diederich, 1995; Miller,

51 1982; Schwarz, 1989, 1994, 2006).

52 We demonstrate that the behavioral benefits accrued by monkeys when detecting vocalizations

53 are a lawful function of both the intensities and the delay between sensory stimuli (Cluff et al., 2015;

54 Crevecoeur et al., 2016; Dixon \& Spitz, 1980; Holmes, 2009; Meredith et al., 1987; Stein et al., 1989;

55 van Wassenhove et al., 2007). After ensuring that separate activation models are insufficient to

56 explain the behavior of the monkeys (Miller, 1986; Raab, 1962) we show that the superposition

57 framework that assumes additivity of visual and auditory inputs and accumulation to a bound 
provides an approximate description of the RTs but fails to describe the accuracy of the monkeys performing the task (Diederich, 1995; Schwarz, 1989, 1994). We, therefore, expanded on the diffusion superposition model by incorporating a deadline for accumulation. We derive closed-form solutions for the mean reaction times (RTs) and accuracy rates for the two-stage diffusion process with drift towards a single barrier in the presence of a temporal deadline. We then show that such an expansion of the classical coactivation model provides an excellent description of both accuracies as well as RTs of these monkeys.

The manuscript is organized as follows. We provide a brief background on the diffusion superposition model. We then describe the details of and results from our behavioral experiments in monkeys and show that the classical model provides an unsatisfactory fit to the accuracy and response times. We then show that extending the model by incorporating a deadline for accumulation provides an better account of the observed behavior of the monkeys. We end by discussing the ramifications of this model.

\section{Redundant Signals Tasks}

The redundant signals task is a simple and powerful experimental paradigm for investigating multisensory perception. In this task, subjects are asked to respond in the same way to stimuli of two sensory modalities, for example, auditory and visual stimuli (A, V, Diederich \& Colonius, 1987; Miller, $1982,1986)$. The typical finding is that if signals from both modalities are present (redundant signals, AV), average responses are faster than average responses to targets from any single modality (unimodal targets). This redundant signals effect, by itself, is not necessarily indicative of any special mechanism that integrates the information of the different channels. Redundancy gains may arise from a race between the channel-specific detection processes ("race model,"Miller, 1982; Raab, 1962). In audiovisual detection, however, redundancy gains are typically larger than predicted by race models and these are thought to be better explained by "coactivation models" that assume some kind of integration of the information provided by the two sensory systems (Diederich, 1995; Diederich \& Colonius, 2004b; Miller, 1986; Schwarz, 1989, 1994). One such model is the diffusion superposition model, which we explain below. 


\section{The diffusion superposition model}

86 The single-barrier diffusion superposition model (Schwarz, 1994) is a coactivation model that describes redundancy gains assuming additive superposition of channel-specific diffusion processes. In diffusion models, the assumption is that the presentation of a stimulus leads to a buildup of evidence that is described by a noisy diffusion process $\mathbf{X}(t)$ with drift $\mu$ and variance $\sigma^{2}>0$ (Ratcliff, 1988; Ratcliff \& McKoon, 2008; Ratcliff, Smith, Brown, \& McKoon, 2016; Smith \& Ratcliff, 2004). The stimulus is 'detected' as soon as an evidence criterion $c>0$ is met for the first time. The density $g(t)$ and distribution $G(t)$ of the first-passage times D are well known ('inverse Gaussian', Cox \& Miller, 1965),

$$
\begin{aligned}
& g\left(t \mid c, \mu, \sigma^{2}\right)=\frac{c}{\sqrt{2 \pi \sigma^{2} t^{3}}} \cdot \exp \left[-\frac{(c-\mu t)^{2}}{2 \sigma^{2} t}\right] \\
& G\left(t \mid c, \mu, \sigma^{2}\right)=\Phi\left(\mu t \mid c, \sigma^{2} t\right)+\exp \left(\frac{2 c \mu}{\sigma^{2}}\right) \Phi\left(-\mu t \mid c, \sigma^{2} t\right)
\end{aligned}
$$

97

with $\Phi\left(x \mid m, s^{2}\right)$ denoting the Normal distribution with mean $m$ and variance $s^{2}$. The expected

$$
E(\mathbf{D})=\frac{c}{\mu}
$$

Predictions for the detection times for unimodal stimuli $\mathrm{A}$ and $\mathrm{V}$ are, therefore, easily obtained, $c / \mu_{\mathrm{A}}$ and $c / \mu_{\mathrm{V}}$, respectively. When two stimuli are presented simultaneously, coactivation occurs. $\mathbf{X}_{\mathrm{V}}(t)$. The new process $\mathbf{X}_{\mathrm{AV}}$ is, therefore, again a diffusion process with drift $\mu_{\mathrm{AV}}=\mu_{\mathrm{A}}+\mu_{\mathrm{V}}$ and variance $\sigma_{\mathrm{AV}}^{2}=\sigma_{\mathrm{A}}^{2}+\sigma_{\mathrm{V}}^{2}+2 \rho_{\mathrm{AV}} \sigma_{\mathrm{A}} \sigma_{\mathrm{V}}$ (under the assumption that $\mathbf{X}_{\mathrm{A}}$ and $\mathbf{X}_{\mathrm{V}}$ are uncorrelated, the covariance term will be zero). Since the drift parameters add up, $\mathbf{X}_{\mathrm{AV}}$ reaches the response criterion earlier than any of its single constituents, resulting in faster responses to redundant stimuli, 
$E\left(\mathbf{D}_{\mathrm{AV}}\right)=\frac{c}{\mu_{\mathrm{A}}+\mu_{\mathrm{V}}}$

What happens in stimuli presented with onset asynchrony (SOA, e.g., V160A, i.e., the visual stimulus component is presented first, and then the auditory stimulus component follows the visual

114 stimulus component with a delay $\tau$, here $160 \mathrm{~ms}$ )? During the first $\tau \mathrm{ms}$, the sensory evidence is

115 accumulated by the visual channel alone. If the criterion is reached within this interval, the stimulus is

116 detected, and a response is initiated. This case occurs with probability

$117 P\left(\mathbf{D}_{\mathrm{V}(\tau) \mathrm{A}} \leq \tau\right)=G\left(\tau \mid c, \mu_{\mathrm{V}}, \sigma_{\mathrm{V}}^{2}\right)$ given by Eq. (2). If detection occurs before a time of $\tau$ has elapsed, it

118 is expected to occur within

$$
E\left(\mathbf{D}_{\mathrm{V}(\tau) \mathrm{A}} \mid \mathbf{D}_{\mathrm{V}(\tau) \mathrm{A}} \leq \tau\right)=\frac{1}{P\left(\mathbf{D}_{\mathrm{V}(\tau) \mathrm{A}} \leq \tau\right)} \int_{0}^{\tau} t \cdot g\left(t \mid c, \mu_{\mathrm{V}}, \sigma_{\mathrm{V}}^{2}\right) d t
$$

$$
w\left(x, \tau \mid c, \mu_{\mathrm{V}}, \sigma_{\mathrm{V}}^{2}\right)=\phi\left(x \mid \mu_{\mathrm{V}} \tau, \sigma_{\mathrm{V}}^{2} \tau\right)-\exp \left(\frac{2 c \mu_{\mathrm{V}}}{\sigma_{\mathrm{V}}^{2}}\right) \cdot \phi\left(x \mid 2 c+\mu_{\mathrm{V}} \tau, \sigma_{\mathrm{V}}^{2} \tau\right),
$$

127 (e.g., Schwarz, 1994, Eq. 7) with $\phi\left(x \mid \mu, \sigma^{2}\right)$ denoting the Normal density with mean $\mu$ and variance $\sigma^{2}$. Because activation level $x$ has already been attained, less 'work' has to be done to reach the aggregate process drifting with $\mu_{\mathrm{AV}}$ towards a residual barrier $c-x$ :

$$
E\left(\mathbf{D}_{\mathrm{V}(\tau) \mathrm{A}} \mid \mathbf{X}(\tau)=x\right)=\tau+\frac{c-x}{\mu_{\mathrm{AV}}}
$$




$$
E\left(\mathbf{D}_{\mathrm{V}(\tau) \mathrm{A}} \mid \mathbf{D}_{\mathrm{V}(\tau) \mathrm{A}}>\tau\right)=\int_{-\infty}^{c} w\left(x, \tau \mid c, \mu_{\mathrm{V}}, \sigma_{\mathrm{V}}^{2}\right) \cdot E\left(\mathbf{D}_{\mathrm{V}(\tau) \mathrm{A}} \mid \mathbf{X}(\tau)=x\right) d x
$$

An analytic solution for the overall, unconditional expected first-passage time $E\left(\mathbf{D}_{\mathrm{V}(\tau) \mathrm{A}}\right)$ has been derived by Schwarz (1994, Eq. 10).

prediction for the observed response time $\mathbf{T}$, an additional variable $\mathbf{M}$ is typically introduced that summarizes everything not described by the diffusion process (e.g., motor execution), such that

where the additional parameter denotes the expectation of M. Schwarz (1994) demonstrated that such a model of additive superposition quite accurately describes the mean response times and variances reported for Participant B.D. from (Miller, 1986) in a speeded response task with 13 different SOAs (at a single intensity).

\section{Present behavioral experiment}

153 Our stated goal is to understand how the benefits in redundant signals tasks depend on both the 154 intensity of the sensory stimuli as well as the SOA. To that end, we trained monkeys to detect visual, 155 auditory and audiovisual vocal signals in a constant background of auditory noise (Chandrasekaran et 156 al., 2013; Chandrasekaran et al., 2011). We chose a free response paradigm (without explicit trial 157 markers, Egan, Greenberg, \& Schulman, 1961; Shub \& Richards, 2009) because it mimics natural 158 audiovisual communication - faces are usually continuously visible and move during vocal production.

159 The task was a typical redundant signals task (Miller, 1982, 1986); stimuli were chosen to 160 approximate natural face-to-face vocal communication. This task was heavily inspired by the observation that besides providing benefits for discrimination of speech sounds (Besle, Fort,

162 Delpuech, \& Giard, 2004; Grant, Walden, \& Seitz, 1998), visual speech enhances the detection of 163 auditory speech (Grant, 2001; Grant \& Seitz, 2000; Schwartz, Berthommier, \& Savariaux, 2004). In 
164 such settings, the vocal components of the communication signals are degraded by environmental

165 noise. The motion of the face, on the other hand, is usually perceived clearly. In the task, monkeys

166 detected the onset of 'coo' calls that are affiliative vocalizations commonly produced by macaque

167 monkeys in a variety of contexts (Hauser \& Marler, 1993; Rowell \& Hinde, 1962). The coo calls were

168 presented at three different levels of sound intensity and were embedded in a constant background

169 noise. The visual signals were videos of monkey avatars opening their mouth to make a coo

170 vocalization. The size of the mouth opening was in accordance with the intensity of the associated

171 vocalization: greater sound intensity was coupled to larger mouth openings by the dynamic face.

172 Finally, we generated audiovisual stimuli by combining the videos with the coo vocalizations. The task

173 of the monkeys was to detect the visual motion of the mouth or the onset of the coo vocalization.

174 Audiovisual stimuli were presented either in synchrony or at 10 different SOAs.

\section{Methods}

\section{Subjects}

177 Nonhuman primate subjects were two adult male macaques ( $\mathrm{S}$ and B, Macaca fascicularis). The

178 monkeys were born in captivity and housed socially. The monkeys underwent sterile surgery for the

179 implantation of a painless head restraint (see Chandrasekaran, Turesson, Brown, \& Ghazanfar, 2010).

180 All experiments and surgical procedures were performed in compliance with the guidelines of the

181 Princeton University Institutional Animal Care and Use Committee.

\section{Procedure}

183 Experiments were conducted in a sound attenuating radio frequency enclosure. The monkey sat in a 184 primate chair fixed $74 \mathrm{~cm}$ opposite a 19 inch CRT color monitor with a $1280 \times 1024$ screen resolution 185 and $75 \mathrm{~Hz}$ refresh rate. The screen subtended a visual angle of $\sim 25^{\circ}$ horizontally and $20^{\circ}$ vertically. All

186 stimuli were centrally located on the screen and occupied a total area (including blank regions) of

$187640 \times 653$ pixels. For every session, the monkeys were placed in a restraint chair and head-posted. A

188 depressible lever (ENV-610M, Med Associates) was located at the center-front of the chair. Both 
189 monkeys spontaneously used their left hand for responses. Stimulus presentation and data collection 190 were performed using Presentation (Neurobehavioral Systems).

\section{Stimuli}

192 Coo vocalizations could be one of three different levels of sound intensity $(85 \mathrm{~dB}, 68 \mathrm{~dB}, 53 \mathrm{~dB})$ and

193 were embedded in a constant background noise of $63 \mathrm{~dB}$ SPL (giving us a range of signal to noise

194 ratios, SNR, Fig. 1A). We used coo calls from two macaques as the auditory components of

195 vocalizations; these were recorded from individuals that were unknown to the monkey subjects. The

196 auditory vocalizations were resized to a constant duration of 400 ms using a MATLAB implementation

197 of a phase vocoder (Flanagan \& Golden, 1966) and normalized in amplitude (Fig. 1A). The visual

198 components of the vocalizations were $400 \mathrm{~ms}$ long videos of synthetic monkey agents making a coo

199 vocalization. The animated stimuli were generated using 3D Studio Max 8 (Autodesk) and Poser Pro

200 (Smith Micro), and were extensively modified from a stock model made available by DAZ Productions

201 (Silver key 3D monkey, Figs. 1B, C). Further details of the generation of these visual avatars are

202 available in a prior study (Chandrasekaran et al., 2011).

The audiovisual stimuli were generated by presenting both the visual and auditory components

204 either in synchrony or at 10 different stimulus onset asynchronies (SOAs). Audition could precede 205 vision by 240, 160, 120, 80 or 40 ms (Fig. 1D) and vice versa (Fig. 1E). Intensities were always paired; 206 that is the weak auditory stimulus was always paired with a small mouth opening. We, therefore, had 2073 pairs of intensities and 11 SOAs, which resulted in 33 AV conditions in total. Each block also had 3 208 auditory intensities, 3 visual intensities. Catch trials (C) were used to discourage from spontaneous responses and to be able to control for fast guesses in the analysis of the RT distributions.

\section{Task}

211 During the task (Fig. 1F), an avatar face (e.g., Avatar 1) was continuously present on the screen; the 212 background noise was also continuous. In the visual-only condition (V), Avatar 1 moved its mouth without any corresponding auditory component. In the auditory-only condition $(A)$, the vocalization

214 paired with Avatar 2 was presented with the static face of Avatar 1. Finally, in the audiovisual condition (AV), Avatar 1 moved its mouth accompanied by the corresponding vocalization of Avatar 1 
216 and in accordance with its intensity. We, therefore, had two AV stimuli. (A1V1 and A2V2). In the even

217 blocks, the avatar face was the still frame of V1, A2 was the auditory sound played, and A1V1 was the

218 audiovisual stimulus. The other block had the opposite configuration. This task design avoids the

219 conflict between hearing a vocalization with the corresponding avatar face not moving.

A

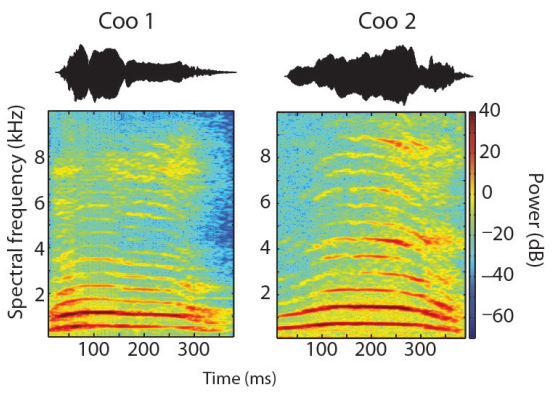

B

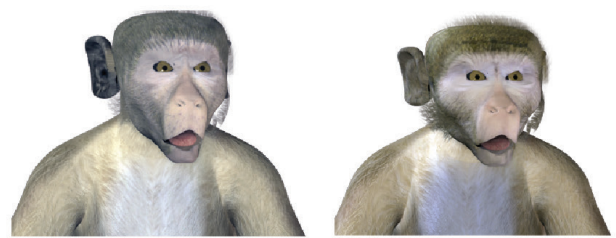

C

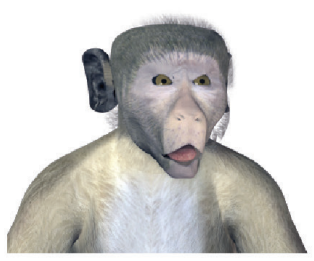

$+22 \mathrm{~dB}$

High

D

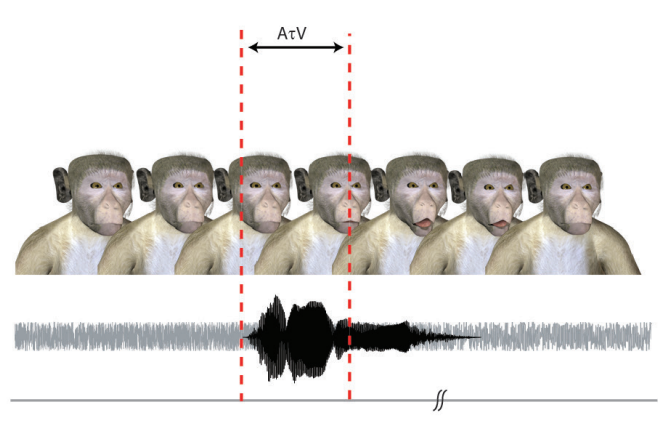

Audition preceding Vision

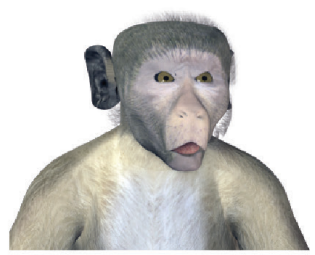

$+5 \mathrm{~dB}$

Medium

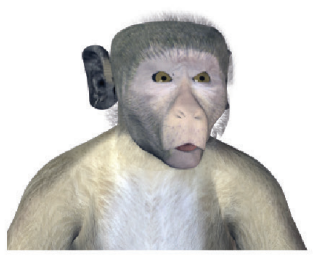

$-10 \mathrm{~dB}$

Low

Figure 1

E

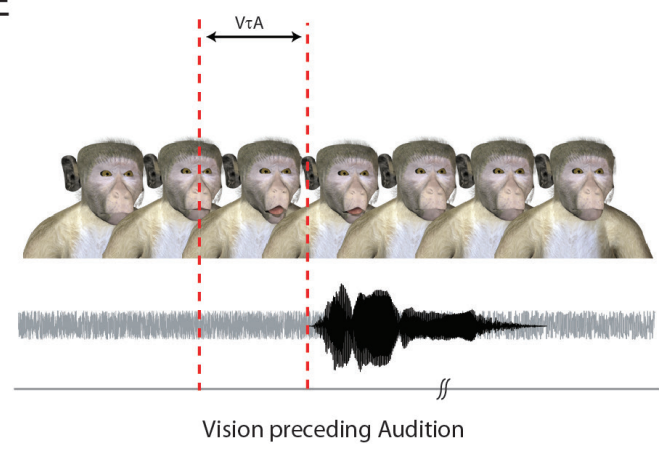

F

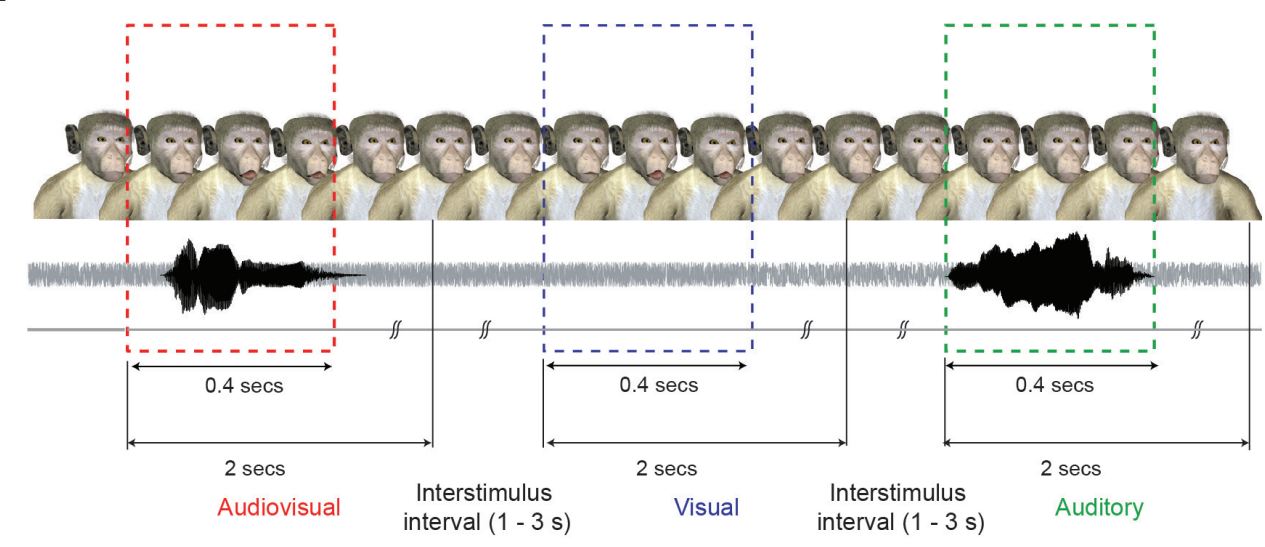

Page 10 
Figure 1 (previous page): Stimuli, task structure, and behavior. A: Waveform and spectrogram of coo vocalizations detected by the monkeys. B: Frames of the two monkey avatars at the point of maximal mouth opening for the largest SNR. C: Frames with maximal mouth opening from one of the monkey avatars for three different SNRs of $+22 \mathrm{~dB}$ (High), $+5 \mathrm{~dB}$ (Medium) and $-10 \mathrm{~dB}$ (Low). D: An illustration of the $A(\tau) \vee$ audiovisual condition. In this stimulus, the onset of the vocalization precedes the onset of the mouth motion. E: An illustration of the $V(\tau) A$ audiovisual condition. In this stimulus, visual cues such as mouth opening precede the onset of the vocalization. F: Task structure for monkeys. An avatar face was always on the screen. Visual, auditory and audiovisual stimuli were randomly presented with an inter stimulus interval of 1-3 seconds drawn from a shifted and truncated exponential distribution. Responses within a $2 \mathrm{sec}$ window after stimulus onset were considered hits. Responses in the interstimulus interval are considered to be response errors and led to timeouts.

Stimuli of each condition $(\mathrm{V}, \mathrm{A}, \mathrm{AV}, \mathrm{C})$ were presented after a variable inter stimulus interval between 1 and 3 seconds (drawn from a shifted and truncated exponential distribution). Monkeys indicated the detection of a V, A or AV event by pressing the lever within 2 seconds following the onset of the stimulus. In the case of hits, the ISI was started immediately following a juice reward. In the case of misses, the ISI began after the two second response window.

After every block of 126 trials ( $33 \mathrm{AV}$ stimuli $\times 3$ trials $+3 \mathrm{~A} \times 3$ trials, $3 \mathrm{~V} \times 3$ trials, 9 catch trials), a brief pause ( 10 to 12 seconds) was imposed. Then, a new block was started in which, the avatar face, and the identity of the coo sound used for the auditory-only condition were switched. Within a block, all the conditions were randomly interleaved with one another.

\section{Training}

230 Monkeys were initially trained over many sessions to respond to the coo vocalization events in visual, auditory or audiovisual conditions while withholding responses when no stimuli were presented. A press of the lever within a window starting $150 \mathrm{~ms}$ after onset of the vocalization event and within two seconds led to a juice reward and was defined as a hit. An omitted response in this two-second window was classified as a miss similar to the studies of free response tasks (without explicit trial markers , Egan et al., 1961; Shub \& Richards, 2009). Lever presses to catch trials were defined as false alarms. In addition, the random presses during the interstimulus interval (ISI) were discouraged by enforcing a timeout where no stimuli were presented. The timeout was chosen randomly from a 
distribution between 3.0 and $5.5 \mathrm{~s}$. The monkeys had to wait for the entire duration of this timeout period before a new stimulus was presented. Any lever press during the timeout period led to a renewal of the timeout with the duration again randomly drawn from the same distribution. Monkeys were trained until the erroneous presses in this ISI period were less than or equal to $10 \%$ of trials in any given session.

\section{Statistical analysis of behavioral performance}

244 Hit rate was defined as the ratio of hits to the total number of targets. For each SNR and condition, 245 the accuracy was defined as the ratio of hits to hits plus misses expressed as a percentage. The false

246 alarm rate (i.e., presses to the catch stimuli) was defined as the number of false alarms divided by the 247 total number of catch trials. Mean RTs and SDs were computed for the correct responses; confidence 248 intervals were obtained by resampling the observed RT distributions (including omitted responses 249 and false alarms) with replacement 1000 times and estimating the standard deviation of the mean of 250 the resampled data.

\section{Test of the race model inequality}

252 An important model class for redundant signals effects is the so-called race model, or separate 253 activation model (Colonius \& Diederich, 2006; Gondan \& Minakata, 2016; Miller, 1982, 1986; Raab, 254 1962). According to the race model, redundancy benefits are not due to an actual integration of visual and auditory signals but because of parallel processing of both signals. In the bimodal stimulus, the two channels engage in a race-like manner ("parallel first-terminating model", Townsend \& Ashby, 1983), so that the probability for fast responses is increased because slow processing times are canceled out by the other channel. The redundancy gains obtained in the race model are limited, however, and a classical test of whether this mechanism can explain the observed reaction times is the well- known race model inequality (Miller, 1982), stating that the RT distribution for redundant stimuli $F_{\mathrm{AV}}(t)$ never exceeds the sum of the RT distributions for the unisensory stimuli $F_{\mathrm{A}}(t), F_{\mathrm{V}}(t)$,

$$
F_{\mathrm{AV}}(t) \leq F_{\mathrm{A}}(t)+F_{\mathrm{V}}(t), \text { for all } t
$$


265 (Eriksen, 1988, see also Gondan and Heckel, 2008) demonstrated that this inequality could be refined 266 by taking into account anticipatory responses to catch trials (C),

267

268

269

270 271

272

273

274

275

276

277

278

279

280

281

282

283

284

285

286

287

288

289

290

$$
F_{\mathrm{AV}}(t)+F_{\mathrm{C}}(t) \leq F_{\mathrm{A}}(t)+F_{\mathrm{V}}(t), \text { for all } t
$$

For redundant targets presented with SOA $\tau$, the inequality generalizes to (Miller, 1986)

$$
F_{\mathrm{V}(\tau) \mathrm{A}}(t)+F_{\mathrm{C}}(t-\tau) \leq F_{\mathrm{V}}(t)+F_{\mathrm{A}}(t-\tau), \text { for all } t
$$

If this inequality is violated in a given data set, then parallel self-terminating processing cannot account for the benefits observed for multisensory stimuli, suggesting an explanation based on the integration of the signals (e.g., the superposition model described above; see also (Luce, 1986; Miller, 2016) for a discussion of the assumption of context independence).

For each condition, we determined the empirical cumulative distribution functions (eCDFs) and then computed the maximum violation, that is, the maximum difference between the left-hand side and the right-hand side of Inequality 11. A bootstrap technique (Miller, 1986), was used to assess the statistical significance of the observed violations of the race model inequality.

\section{Test of the diffusion superposition model}

We fit the predictions of the diffusion superposition model to the mean RTs from the two monkeys. We used the analytic equation provided in Schwarz (1994, Eq. 10). To perform model fitting, we computed for each monkey, an approximate $\chi_{26}^{2}$ goodness-of-fit statistic given by the sum of the squared standardized deviations of the predicted and the observed average response times (e.g., Schwarz, 2006). The 26 degrees of freedom are given by the difference between the number of conditions ( 3 intensities $\times 13$ SOAs) minus the number of model parameters: 12 parameters are due to the drift and variance for each visual and auditory SNR ( 3 SNRs $\times 2$ modalities $\times 2$ parameters). The thirteenth parameter is the average residual non-decision time (Eq. 8). 


\section{Results}

292 Figures 2A, C show the accuracy of the two monkeys in the detection task for different SNRs and 293 SOAs. The accuracy (see Figure 2A, C, see also Supplementary Tables S1-S4) showed lawful decreases 294 with changes in SNR for the auditory components of vocalizations (e.g., only about 55\% detection rate 295 with auditory stimuli). In contrast, changes in mouth opening size for the visual component of the vocalizations, which were meant to match the changes in auditory intensity, had only minimal impact on the accuracy of the animals. The mean RTs (Figures 2B,D) showed the wing shaped pattern typically observed in redundant signals tasks with SOA manipulations (Miller, 1986; Ulrich \& Miller, 1997).
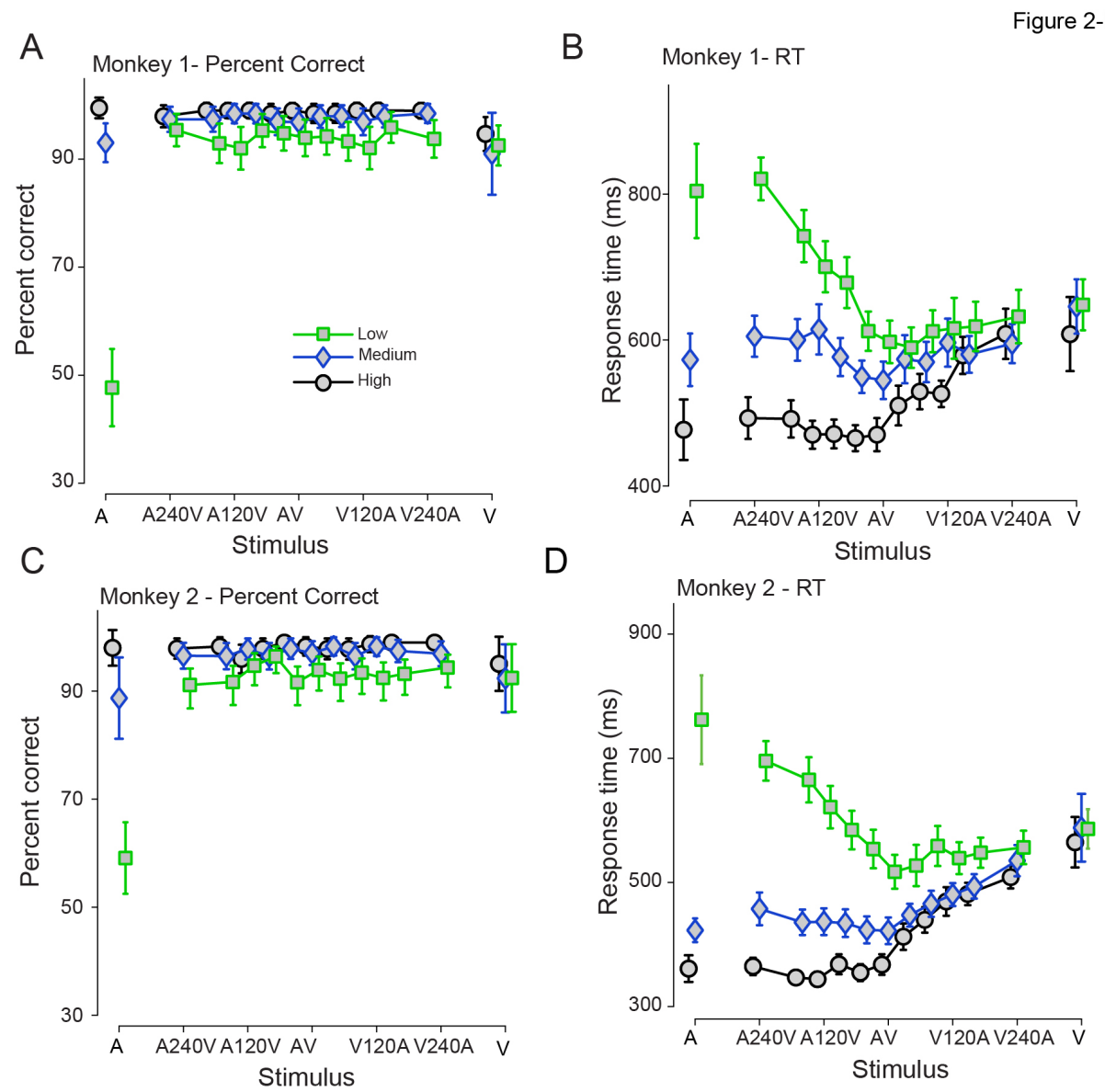

Figure 2: Monkeys integrate visual and auditory cues across a large range of SOAs and SNRs

A, B: Mean accuracy and RTs for audiovisual, visual-only and auditory-only conditions for Monkey 1 ( $A$, B) and Monkey 2 (C, D ) as a function of different stimulus conditions and SNR. X-axes depict stimuli. Yaxes in the left panel depict the accuracy in percent. $Y$-axes in the right panel denote response times in ms. Error bars denote confidence intervals around the mean $(2 \times \mathrm{SEM})$. 
In both monkeys, statistically significant violations of the upper bound of the race model

302 inequality for RTs (Inequality 11) occurred for a large range of SOAs, indicating that the observed

303 redundancy gains were inconsistent with a race model. For Monkey 1, statistically significant

304 violations of the RMI (at the criterion of $P<0.05$ ) were observed in 28 out of the 33 multisensory

305 conditions, for Monkey 2, this was observed in all 33 conditions (Tables S5, S6 in online supplement).

306

We first examined if the diffusion superposition model could describe the behavior of the

308 monkeys during this task. We found that the superposition model could describe the overall pattern

309 of the mean RTs of the monkeys (Figures 3A, B). However, the overall fit was unsatisfactory and

310 especially poor for the lowest SNRs (Monkey $1: \chi_{26}^{2}=49.2, P=0.004$; Monkey $2: \chi_{26}^{2}=53.9, P=$

A

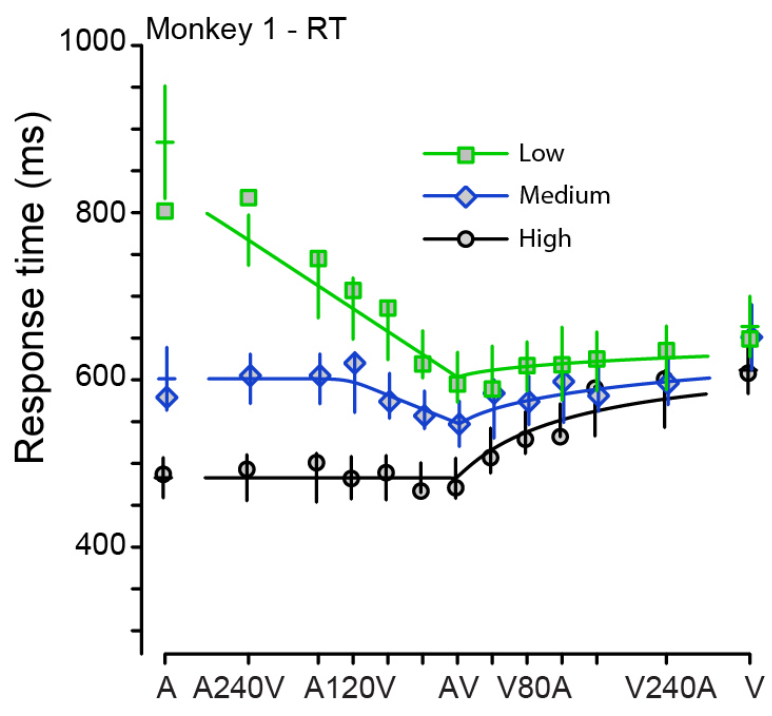

Conditions
B

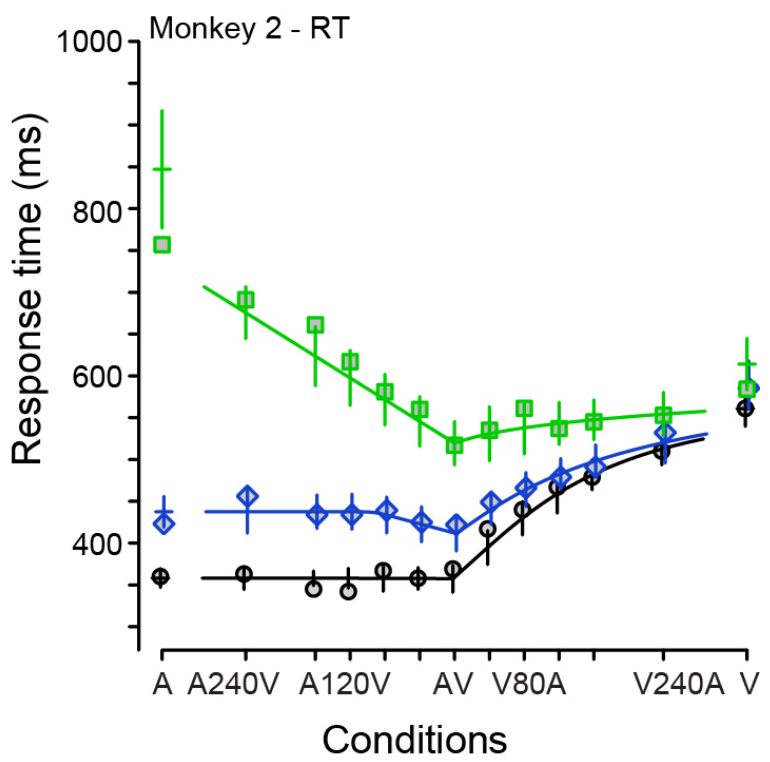

Figure 3: The diffusion superposition model provides an incomplete description of the detection behavior of monkeys

Audiovisual, visual-only and auditory-only RTs for both monkeys along with the predicted RT shown in lines according to the diffusion superposition model as a function of SNR (squares = low , diamonds $=$ medium , circles $=$ high) and SOA. Error bars denote confidence intervals $(2 \times$ standard error) around predicted mean RTs. A shows the RT for monkey 1; B shows the RT for monkey 2. 
311 0.001). The monkeys also omitted a substantial proportion of responses to weak auditory stimuli

312 (detection accuracy rates for the lowest auditory intensity were $~ 55 \%$ and $60 \%$ ), which is, by design,

313 not accounted for by the superposition model described above. The integral in Equation 3 ranges

314 from 0 to infinity, such that, absorption at the upper barrier is a certain event given enough time. This

315 means that the superposition model always predicts ceiling level accuracies for all intensities, a

316 prediction clearly inconsistent with the observed behavioral data.

\section{A coactivation model with a deadline}

318 An unrealistic assumption of the model described above is that accumulation will always complete, 319 which in the single-barrier diffusion model implies that the monkeys have 100 percent detection 320 accuracy. Given enough time, a diffusion process with drift $\mu>0$ will almost certainly reach the 321 criterion. From an experimental perspective, this has several implications: the intensity of the 322 stimulus components must be sufficiently high to ensure detection rates of $100 \%$ and the temporal 323 window for responding is infinitely long to guarantee that all responses are collected.

324 However, if the temporal window for stimulus detection is limited by a deadline $d$ (we assume that $325 d>\tau)$ the proportion of correct responses is given by the distribution of the detection times at $t=$ $326 d$. For unimodal stimuli and synchronous audiovisual stimuli, this probability corresponds to the 327 inverse Gaussian distribution at time $d, P\left(\mathbf{D}_{i} \leq d\right)=G\left(d \mid c, \mu_{i}, \sigma_{i}^{2}\right)$, with $i \in\{\mathrm{A}, \mathrm{V}, \mathrm{AV}\}$, depending 328 on the modality. The expected detection time, conditional on stimulus detection before $d$, is again 329 obtained by integration of $t \cdot g(t)$ from $t=0$ to $d$ (see Eq. 4). The solution has been originally 330 presented in (Schwarz, 1994, Eq. 6)

332 In bimodal stimuli with onset asynchrony $0<\tau<d$ (say, without loss of generality, $\mathrm{V}(\tau) \mathrm{A}$ ), it is 333 necessary to distinguish the intervals $[0 \ldots \tau]$ and $[\tau \ldots d]$ during which the drift (the variance) of the 334 diffusion process amount to $\mu_{\mathrm{V}}\left(\sigma_{\mathrm{V}}^{2}\right)$ and $\mu_{\mathrm{AV}}\left(\sigma_{\mathrm{AV}}^{2}\right)$, respectively. The probability for correct 335 detections amounts to the sum of the detections within $[0 \ldots \tau]$ in which only the first stimulus 336 contributes to the buildup of evidence; and the detections within $[\tau \ldots d]$ in which both stimuli are 337 active. 


$$
\begin{aligned}
& P\left(\mathbf{D}_{\mathrm{V}(\tau) \mathrm{A}} \leq d\right)=P\left(\mathbf{D}_{\mathrm{V}(\tau) \mathrm{A}} \leq \tau\right)+P\left(\tau<\mathbf{D}_{\mathrm{V}(\tau) \mathrm{A}} \leq d\right) \\
& \quad=P\left(\mathbf{D}_{\mathrm{V}} \leq \tau\right)+\int_{-\infty}^{c} P[\mathbf{X}(\tau)=x] \cdot P\left(\mathbf{D}_{\mathrm{AV}} \leq d-\tau \mid \mathbf{X}(\tau)=x\right) d x \\
& \quad=G\left(\tau \mid c, \mu_{\mathrm{V}}, \sigma_{\mathrm{V}}^{2}\right)+\int_{-\infty}^{c} w\left(x, \tau \mid c, \mu_{\mathrm{V}}, \sigma_{\mathrm{V}}^{2}\right) \cdot G\left(d-\tau \mid c-x, \mu_{\mathrm{AV}}, \sigma_{\mathrm{AV}}^{2}\right) d x(12)
\end{aligned}
$$

342

343 with $P[\mathbf{X}(\tau)=x]=w(x, \tau \mid \cdots)$ denoting the density of the activation of the processes not yet

344 absorbed at $t=\tau$ (Eq. 5). The integrand decomposes into a sum of four terms of the form $\phi(x)$.

$345 \Phi\left(x \mid m, s^{2}\right)$ that can be integrated using the bivariate Normal distribution (Owen, 1980, Eq.

346 10,010.1, see Appendix A). For the predicted amount of correct responses, a $\chi_{26}^{2}$ statistic is obtained

347 (e.g.,Schwarz, 2006) by the squared difference between the predicted and observed proportion of

348 responses, divided by the variance for binomial proportions $\pi(1-\pi) / N$, with $\pi=P(\mathbf{D} \leq d)$

349 denoting the probabilities for correct detections. To avoid numerical problems close to zero or one, $\pi$ 350 was bounded within [0.01, 0.99].

$$
\begin{aligned}
& E\left(\mathbf{D}_{\mathrm{V}(\tau) \mathrm{A}} \mid \mathbf{D}_{\mathrm{V}(\tau) \mathrm{A}} \leq d\right)=\frac{1}{P\left(\mathbf{D}_{\mathrm{V}(\tau) \mathrm{A}} \leq d\right)} \times \\
& {\left[P\left(\mathbf{D}_{\mathrm{V}(\tau) \mathrm{A}} \leq \tau\right) \cdot E\left(\mathbf{D}_{\mathrm{V}(\tau) \mathrm{A}} \mid \mathbf{D}_{\mathrm{V}(\tau) \mathrm{A}} \leq \tau\right)+P\left(\tau<\mathbf{D}_{\mathrm{V}(\tau) \mathrm{A}} \leq d\right)\right.} \\
& \left.E\left(\mathbf{D}_{\mathrm{V}(\tau) \mathrm{A}} \mid \tau<\mathbf{D}_{\mathrm{V}(\tau) \mathrm{A}} \leq d\right)\right]
\end{aligned}
$$

The second term is more complicated, 
The $w \cdot G$ term corresponds to Equation 12, multiplied by $\tau$. The double integral decomposes into

$$
E(\mathbf{T})=E(\mathbf{D})+\mu_{\mathrm{M}}
$$

For each monkey, an approximate $\chi_{25}^{2}$ goodness-of-fit statistic can be determined by the sum of the squared standardized deviations of the predicted and the observed average response times (e.g.,Schwarz, 2006). Compared to the model without deadline, one degree of freedom is lost because the deadline is adjusted to the data. Because the $\chi^{2}$ statistics for the mean RTs and criterion.

\section{Results for the deadline model}

Figure 4 show the results from fitting the diffusion superposition model with the deadline to the behavioral performance of the monkeys (accuracy and mean RTs) as a function of the signal-to-noise 
391 ratio and the SOAs. The additional deadline parameter improves the model fits and provides a very 392 good description of both accuracy and RTs of the monkeys. For Monkey 1 the model provided an 393 excellent fit to the data. (Accuracy: $\chi_{26}^{2}=34.15$; mean RT: $\chi_{25}^{2}=32.99, P_{\min }=0.131$ ). In Monkey 3942 , the model fit was less convincing (Accuracy: $\chi_{26}^{2}=64.63$; mean RT: $\chi_{25}^{2}=63.08, P_{\min }<0.001$ ), 395 but still acceptable given the conservative fitting procedure where we try to jointly fit both the RTs 396 and accuracy of the monkeys. The best fit parameter estimates are shown in Table 1.

Table 1. Model parameters

\begin{tabular}{lcc}
\hline Parameter & Monkey 1 & Monkey 2 \\
\hline$\mu_{\mathrm{A}}$ (low, medium, high) & $0.01,0.25,1.53$ & $0.09,0.41,9.74$ \\
$\sigma_{\mathrm{A}}^{2}$ (low, medium, high) & $18.5,130.3,74.4$ & $11.7,379.6,10.7$ \\
$\mu_{\mathrm{V}}$ (low, medium, high) & $0.35,0.37,0.34$ & $0.30,0.34,0.37$ \\
$\sigma_{\mathrm{V}}^{2}$ (low, medium, high) & $73.2,68.0,92.8$ & $67.8,73.1,41.9$ \\
$\mu_{\mathrm{M}}$ (msec) & 419 & 343 \\
Deadline $d$ (msec) & 951 & 828 \\
\hline
\end{tabular}


A

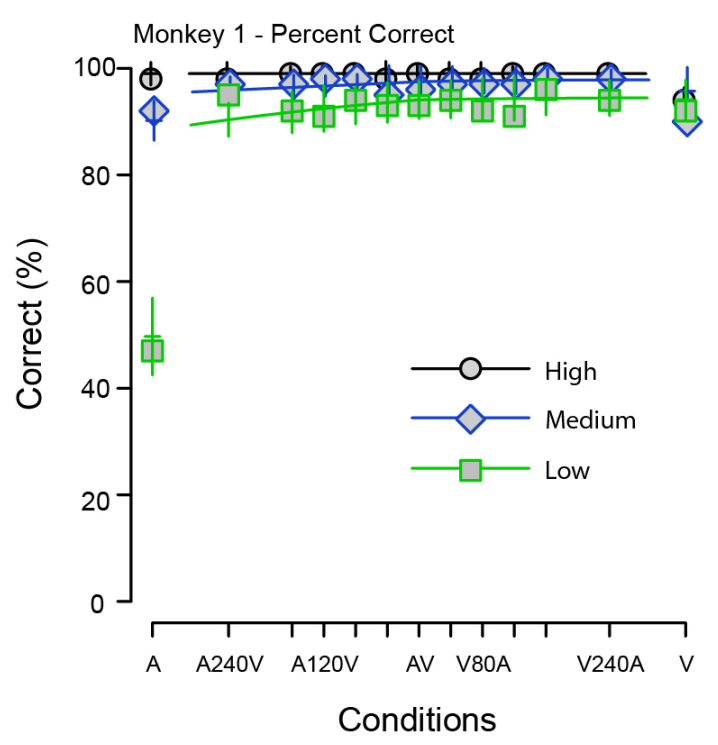

C

Monkey 2 - Percent Correct

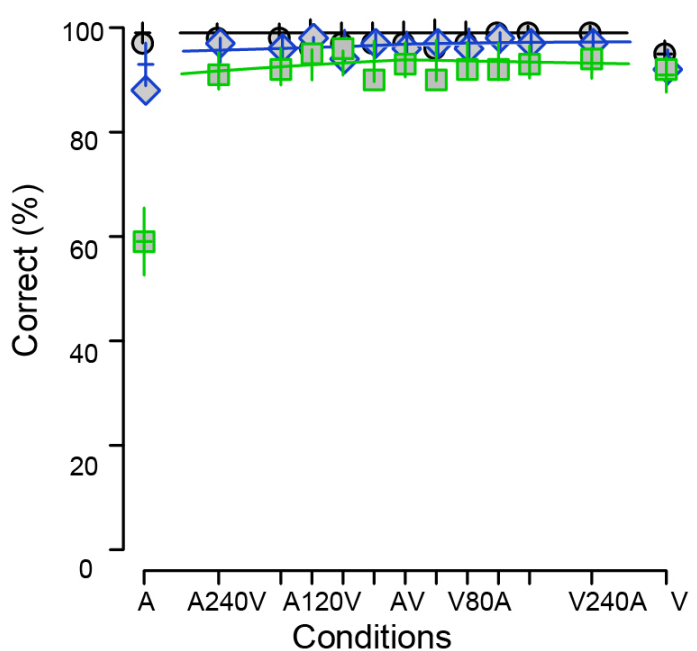

B

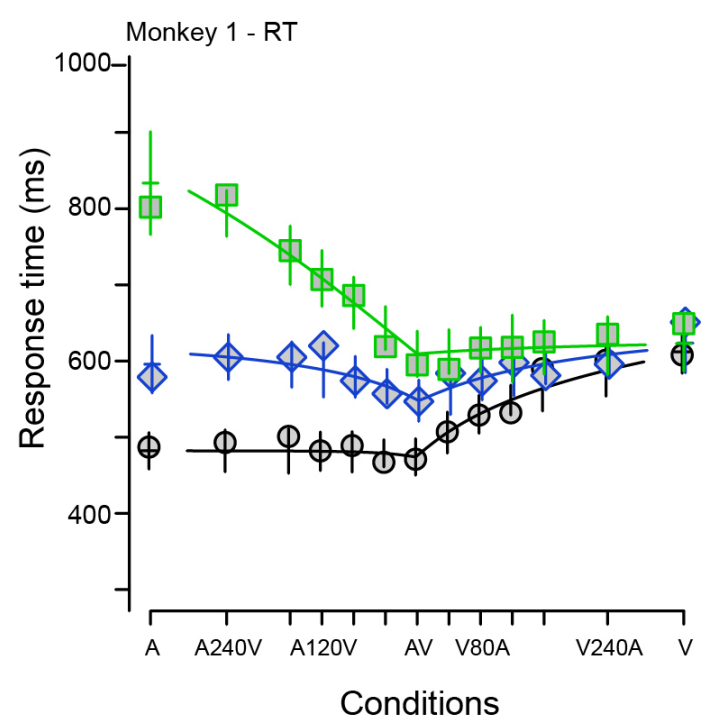

D

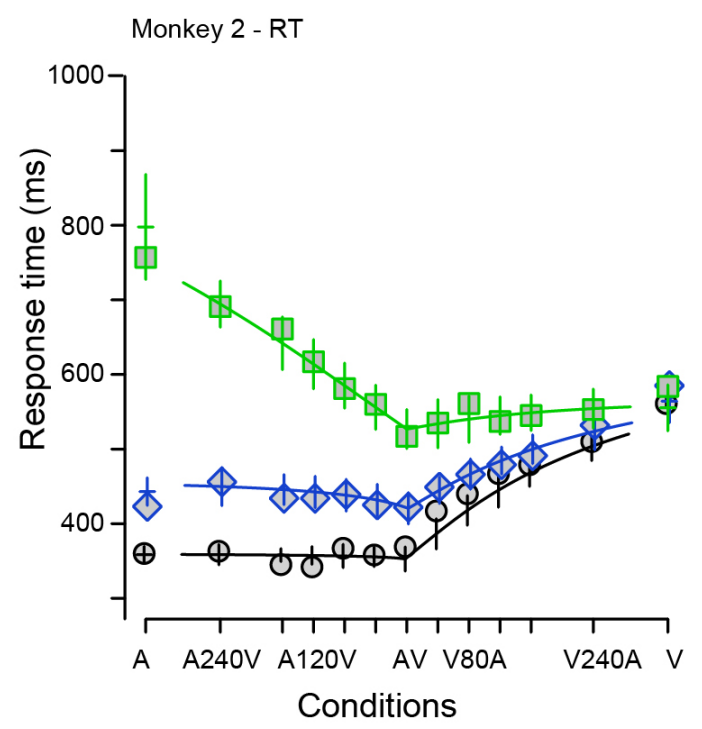

Figure 4: A diffusion superposition model with a time deadline predicts monkey RTs and response

Accuracy and RT of the two monkeys along with the fits from the diffusion superposition model with a deadline. A, C Accuracy of the monkeys as a function of SNR and SOA. X-axes show SOA; Y-axes show the percent correct. B, D Response time of the monkeys as a function of SNR And SOA. X-axes show SOA; Y-axes show Response Time in ms. In both panels, the high SNRS are shown in black circles, the 406 medium SNRs in blue diamonds and low SNRs in green squares. 


\section{Discussion}

408 The goal of our study was to test if the single-barrier diffusion superposition model (Schwarz, 1989, 409 1994; Diederich, 1995) can describe accuracy and RTs to audiovisual vocalizations of different 410 intensities in a detection task. In the auditory modality the intensity manipulation was effective 411 (Figures 2 and 3). In line with this, the drift estimates $\mu_{\mathrm{A}}$ monotonically increased with SNR (Table 1; 412 the variance estimates showed a less regular pattern). In the visual modality, drift estimates $\mu_{\mathrm{V}}$ and 413 variance estimates $\sigma_{\mathrm{V}}^{2}$ were more or less equal for the different intensities, which is consistent with 414 the converging pattern of the mean RTs for positive SOAs (Figure 2). The residual $\mu_{\mathrm{M}}$ was similar in 415 the two animals, reflecting their overall response speed and the fact that stimulus detection is 416 probably just one of several stages of the overall response process.

417 Consistent with many earlier results in bimodal divided attention (Diederich \& Colonius, 2004a; 418 Molholm et al., 2002; Murray et al., 2005), separate activation (a.k.a. race) models were insufficient 419 to explain the behavioral patterns we observed (Miller, 1982, 1986). We used many SOA conditions 420 and thus the majority of the stimuli used in the present study were audiovisual stimuli. Enrichment of 421 audiovisual conditions rules out trial history effects and modality shift effects as the only driving force 422 of coactivation effects (Gondan, Lange, Rösler, \& Röder, 2004; Miller, 1986; Otto, Dassy, \& 423 Mamassian, 2013; Otto \& Mamassian, 2012).

424 We have focused on describing and modeling the mean RTs and mean response accuracy for a 425 detection task across different SNRs and SOAs (Cluff et al., 2015; Crevecoeur et al., 2016; Dixon \& 426 Spitz, 1980; Holmes, 2009; Meredith et al., 1987; Stein et al., 1989; van Wassenhove et al., 2007).

427 Some studies have addressed the effect of sensory reliability which is related to the sensory intensity 428 manipulation we performed here on benefits of multisensory integration but did not modulate the 429 delay between the sensory stimuli (Drugowitsch, DeAngelis, Klier, Angelaki, \& Pouget, 2014). Other 430 studies have examined the dependence on sensory delays but not on sensory intensity (Crevecoeur et 431 al., 2016). Experiments that simultaneously vary stimulus intensity, stimulus reliability and SOA are 432 needed to fully understand the relative roles of these factors in multisensory discrimination(Gondan 433 et al., 2010) . 
Our study is focused on describing the behavior of monkeys performing an audiovisual

detection task. Our model is likely to apply to humans performing similar tasks. We previously showed that the behavior of monkeys and humans in a simpler version of this task that only involved variation in intensity of the sensory stimuli was very similar (Chandrasekaran et al., 2011). Our analysis here expands on a classical coactivation model which has been previously used to successfully model the behavior of a human participant across a range of SOAs (Schwarz, 1994).

We have shown that an accumulator, which integrates visual and auditory inputs to a bound, 441 explained the behavioral benefits from multisensory integration. However, in our task design, no 442 explicit trial onset information was provided to the animals. Instead, the stimulus arrived in a 443 continuous ongoing stream. This paradigm has several advantages because it mimics a natural flow of 444 stimuli in the real world and avoids sharp transients in visual stimuli. But, it raises the important 445 question of how an integrator knows when to begin integrating the sensory evidence? One plausible 446 solution is that a neural circuit resets the integrator after either the last behavioral action by the 447 animal (false alarm/correct detection) or after some time has elapsed (Janssen \& Shadlen, 2005). The 448 fits may improve by incorporating previous ideas which propose to jointly model the inter stimulus 449 interval as well as the responses to sensory stimuli.

450 The superposition model with a deadline predicts RTs and accuracy of monkeys when they 451 detect dynamic visual and auditory stimuli (vocalizations). In other contexts, generalized variants of 452 these coactivation models have been used with dynamic stimuli (Drugowitsch et al., 2014). We 453 believe these types of models may also be applicable to static audiovisual stimuli for two reasons. 454 First, Diffusion models are commonly used with static visual stimuli (Ratcliff, Thapar, \& McKoon, 455 2003; Voss, Rothermund, \& Brandtstädter, 2008). Second,the superposition model has been used to 456 explain discrimination behavior for static audiovisual stimuli (Gondan et al., 2010; Schwarz, 1989, 457 1994). Applying these models to both static and dynamic multisensory stimuli in the same study may 458 help test proposals that there are different mechanisms for the processing of static and dynamic 459 multisensory stimuli (Raposo, Sheppard, Schrater, \& Churchland, 2012). 
461 The key contribution was to show that a model with additive superposition of the channel-specific

462 evidence explains the benefits of integrating faces and voices in animal perception across a wide

463 range of SOAs and SNRs. This class of coactivation models has previously been used to explain

464 response times of human participants in auditory-visual detection tasks (Diederich, 1995; Schwarz,

$4651989,1994)$. The emphasis of these additive coactivation models (or more general versions, e.g.,

466 (Drugowitsch et al., 2014)) seems prima facie at odds with classical reports promoting superadditive

467 multisensory interaction (Stein \& Meredith, 1993). In these studies, superadditivity, and other

468 nonlinear mechanisms were considered fundamental for mediating benefits from multisensory

469 integration. However, as a series of studies have shown, the majority of neurons in classical

470 multisensory brain regions such as the superior colliculus accumulate their synaptic input in a linear

471 manner for a range of stimulus intensities, and nonlinearities are observed only at very low intensities

472 (Dahl, Logothetis, \& Kayser, 2010; Populin \& Yin, 2002; Skaliora, Doubell, Holmes, Nodal, \& King,

473 2004; Stanford, Quessy, \& Stein, 2005; Stanford \& Stein, 2007; Stein \& Stanford, 2008) . Stated

474 differently, additive combination is the norm. For conflicting stimuli (e.g., in temporal order

475 judgment, where participants are asked to report which modality came first), linear summation may

476 occur in the other direction, with the overall evidence corresponding to the difference between the

477 channel-specific activations (Schwarz, 2006).

478

479 Besides linearity of multisensory integration in single neurons, studies increasingly demonstrate that

480 ensembles of neurons (which might encode stimuli nonlinearly at the single neuron level) can

481 perform linear computations (Ma, Beck, Latham, \& Pouget, 2006). We believe that our abstract

482 behavioral model might be implemented by adopting frameworks such as probabilistic population

483 codes. For example, computationally, at the population level, linear summation of neural activation is

484 possible and yields optimal solutions for a very general class of computational problems (Beck et al.,

485 2008; Ma et al., 2006). Extensions of this model showed that assuming Poisson-like distributions of

486 spike counts allows biological networks to accumulate evidence while choosing the most likely action

487 (Beck et al., 2008). We believe our description of behavioral data by this linear summation model will

488 assist in relating neurophysiological and modeling studies of multisensory detection and broadly 
489 integration (Chandrasekaran, 2016; Fetsch, DeAngelis, \& Angelaki, 2013; Ma et al., 2006; Seilheimer

490 et al., 2014).

\section{Acknowledgments}

492 This work was supported by the National Institutes of Health (NINDS) R01NS054898 provided to Prof.

493 Asif A. Ghazanfar (Princeton University). The experimental work was performed under his auspices in

494 his lab. CC was supported by the Charlotte Elizabeth Procter and Centennial Fellowships from

495 Princeton University. We thank Lauren Kelly for expert care of our monkey subjects, Shawn

496 Steckenfinger for the creation of monkey avatars and Luis Lemus for assistance in collecting

497 behavioral data from the monkeys. CC was supported by K99-NS092972 during the writing of this

498 manuscript.

\section{Supplemental material}

500 The online supplement to this article includes mean RTs and accuracy rates for the two animals

501 (Tables S1-S4), bootstrap test of the race model (Tables S5-S6), as well as a commented R script with

502 an implementation of the deadline model.

503

504

505

506

507 


\section{Tables S1-S6}

509

510

511

512

513

514

515

516

517

518

519

520

Table S1. Mean accuracy and bootstrap standard error for different conditions for Monkey 1

\begin{tabular}{|l|c|c|c|c|c|c|c|c|c|c|c|c|c|}
\hline & A & A240V & A160V & A120V & A80V & A40V & AV & V40A & V80A & V120A & V160A & V240A & V \\
\hline High & $98(1)$ & $98(1)$ & $99(1)$ & $99(1)$ & $99(1)$ & $98(1)$ & $99(1)$ & $98(1)$ & $98(1)$ & $99(1)$ & $99(1)$ & $99(1)$ & $94(2)$ \\
\hline Medium & $92(2)$ & $97(1)$ & $97(1)$ & $98(1)$ & $98(1)$ & $95(2)$ & $96(1)$ & $97(1)$ & $97(1)$ & $97(1)$ & $98(1)$ & $98(1)$ & $90(2)$ \\
\hline Low & $47(4)$ & $95(2)$ & $92(2)$ & $91(2)$ & $94(2)$ & $93(2)$ & $93(2)$ & $94(2)$ & $92(2)$ & $91(2)$ & $96(2)$ & $94(2)$ & $92(2)$ \\
\hline
\end{tabular}

Table S2. Mean RT and Standard Error of RTs for different conditions for Monkey 1.

\begin{tabular}{|l|c|c|c|c|c|c|c|c|c|c|c|c|c|}
\hline & A & A240V & A160V & A120V & A80V & A40V & AV & V40A & V80A & V120A & V160A & V240A & V \\
\hline High & $487(12)$ & $493(14)$ & $501(15)$ & $482(13)$ & $489(14)$ & $467(9)$ & $471(12)$ & $507(14)$ & $529(13)$ & $532(10)$ & $590(15)$ & $601(17)$ & $608(14)$ \\
\hline Medium & $579(19)$ & $605(15)$ & $605(15)$ & $620(18)$ & $574(14)$ & $557(12)$ & $547(14)$ & $584(18)$ & $574(15)$ & $598(17)$ & $581(14)$ & $596(14)$ & $651(20)$ \\
\hline Low & $802(34)$ & $818(15)$ & $745(20)$ & $707(19)$ & $686(17)$ & $619(15)$ & $595(15)$ & $589(15)$ & $617(15)$ & $618(22)$ & $625(18)$ & $635(20)$ & $649(19)$ \\
\hline
\end{tabular}

Table S3. Mean accuracy and bootstrap standard error for different conditions for Monkey 2

\begin{tabular}{|l|c|c|c|c|c|c|c|c|c|c|c|c|c|}
\hline & A & A240V & A160V & A120V & A80V & A40V & AV & V40A & V80A & V120A & V160A & V240A & V \\
\hline High & $97(1)$ & $98(1)$ & $98(1)$ & $96(1)$ & $97(1)$ & $97(1)$ & $97(1)$ & $96(1)$ & $97(1)$ & $99(1)$ & $99(1)$ & $100(0)$ & $95(1)$ \\
\hline Medium & $88(2)$ & $97(1)$ & $96(1)$ & $98(1)$ & $94(2)$ & $97(1)$ & $96(1)$ & $97(1)$ & $96(1)$ & $98(1)$ & $97(1)$ & $97(1)$ & $92(2)$ \\
\hline Low & $59(3)$ & $91(2)$ & $92(2)$ & $95(1)$ & $96(1)$ & $90(2)$ & $93(2)$ & $90(2)$ & $92(2)$ & $92(2)$ & $93(2)$ & $94(2)$ & $92(2)$ \\
\hline
\end{tabular}

Table S4. Mean RT and Standard Error of RTs for different conditions for Monkey 2.

\begin{tabular}{|l|c|c|c|c|c|c|c|c|c|c|c|c|c|}
\hline & A & A240V & A160V & A120V & A80V & A40V & AV & V40A & V80A & V120A & V160A & V240A & V \\
\hline High & $360(6)$ & $363(7)$ & $345(4)$ & $342(6)$ & $367(8)$ & $358(7)$ & $369(8)$ & $417(10)$ & $440(10)$ & $467(12)$ & $479(9)$ & $510(10)$ & $561(11)$ \\
\hline Medium & $423(10)$ & $456(13)$ & $434(10)$ & $434(11)$ & $439(11)$ & $425(11)$ & $422(11)$ & $449(9)$ & $466(10)$ & $479(9)$ & $491(10)$ & $532(13)$ & $585(15)$ \\
\hline Low & $757(36)$ & $691(16)$ & $661(18)$ & $617(17)$ & $581(16)$ & $560(15)$ & $517(13)$ & $535(17)$ & $561(16)$ & $537(13)$ & $545(12)$ & $553(13)$ & $584(16)$ \\
\hline
\end{tabular}

Table S5. $P$ values for race model test (Monkey 1 )

\begin{tabular}{|l|c|c|c|c|c|c|c|c|c|c|c|}
\hline & A240V & A160V & A120V & A80V & A40V & AV & V40A & V80A & V120A & V160A & V240A \\
\hline High & 0.057 & 0.062 & 0.014 & 0.039 & 0.038 & 0.031 & 0.018 & 0.000 & 0.000 & 0.000 & 0.000 \\
\hline Medium & 0.125 & 0.013 & 0.029 & 0.062 & 0.293 & 0.002 & 0.000 & 0.000 & 0.000 & 0.000 & 0.000 \\
\hline Low & 0.043 & 0.000 & 0.000 & 0.000 & 0.000 & 0.001 & 0.000 & 0.040 & 0.000 & 0.007 & 0.000 \\
\hline
\end{tabular}

Table S6. $P$ values for race model test for Monkey 2

\begin{tabular}{|l|c|c|c|c|c|c|c|c|c|c|c|}
\hline & A240V & A160V & A120V & A80V & A40V & AV & V40A & V80A & V120A & V160A & V240A \\
\hline High & 0.001 & 0.001 & 0.000 & 0.004 & 0.005 & 0.033 & 0.000 & 0.000 & 0.000 & 0.000 & 0.000 \\
\hline Medium & 0.008 & 0.003 & 0.011 & 0.041 & 0.000 & 0.000 & 0.004 & 0.000 & 0.000 & 0.000 & 0.000 \\
\hline Low & 0.019 & 0.001 & 0.000 & 0.000 & 0.000 & 0.000 & 0.000 & 0.000 & 0.000 & 0.001 & 0.000 \\
\hline
\end{tabular}




\section{Appendix A: Probability of absorption}

522 Here we derive the explicit expressions for the probability of absorption in bimodal stimuli with onset

523

524

525

526

527

528

529

530

531

532

533

534

535

536

537

538

539

540

541

542

543

544

545

asynchrony $0<\tau<d$ (Eq. 12). Without loss of generality, we consider the case $\mathrm{V}(\tau) \mathrm{A}$ in which the visual stimulus is presented first. Between $t=0$ and $t=\tau$, only the visual channel contributes to the build-up of evidence, so the probability of absorption within the interval $(0, \tau)$ is given by

$$
P\left(\mathbf{D}_{\mathrm{V}(\tau) \mathrm{A}} \leq \tau\right)=G\left(\tau \mid c, \mu_{\mathrm{V}}, \sigma_{\mathrm{V}}^{2}\right)
$$

with $G$ denoting the inverse Gaussian distribution (Eq. 2). Later, within the time interval $(\tau, d)$, the probability of absorption is the mixture of absorption probabilities of those processes still active at time $\tau$, with the barrier depending on the activation $\mathbf{X}(\tau)<c$, weighted by the density of processes at $\mathbf{X}(\tau)$. Let $d^{\prime}=d-\tau$. Then,

$$
P\left(\tau<\mathbf{D}_{\mathrm{V}(\tau) \mathrm{A}} \leq d\right)=\int_{-\infty}^{c} w\left(x, \tau \mid c, \mu_{\mathrm{V}}, \sigma_{\mathrm{V}}^{2}\right) \cdot G\left(d^{\prime} \mid c-x, \mu_{\mathrm{AV}}, \sigma_{\mathrm{AV}}^{2}\right) d x
$$

with $w$ given by Equation 5. The integrand in (A.2) can be transformed into four integrals of the form

$q \int_{-\infty}^{c} \exp (r x) \phi\left(x \mid m_{1}, s_{1}^{2}\right) \cdot \Phi\left(x \mid m_{2}, s_{2}^{2}\right) d x:$

$$
\begin{aligned}
& \int_{-\infty}^{c} w\left(x, \tau \mid c, \mu_{\mathrm{V}}, \sigma_{\mathrm{V}}^{2}\right) \cdot G\left(d^{\prime} \mid c-x, \mu_{\mathrm{AV}}, \sigma_{\mathrm{AV}}^{2}\right) d x \\
& =\int_{-\infty}^{c} \phi\left(x \mid \mu_{\mathrm{V}} \tau, \sigma_{\mathrm{V}}^{2} \tau\right) \Phi\left(x \mid c-\mu_{\mathrm{AV}} d^{\prime}, \sigma_{\mathrm{AV}}^{2} d^{\prime}\right) d x \\
& \quad+\exp \left(\frac{2 c \mu_{\mathrm{AV}}}{\sigma_{\mathrm{AV}}^{2}}\right) \int_{-\infty}^{c} \exp \left(-\frac{2 \mu_{\mathrm{AV}}}{\sigma_{\mathrm{AV}}^{2}} x\right) \phi\left(x \mid \mu_{\mathrm{V}} \tau, \sigma_{\mathrm{V}}^{2} \tau\right) \Phi\left(x \mid c+\mu_{\mathrm{AV}} d^{\prime}, \sigma_{\mathrm{AV}}^{2} d^{\prime}\right) d x \\
& \quad-\exp \left(\frac{2 c \mu_{\mathrm{V}}}{\sigma_{\mathrm{V}}^{2}}\right) \int_{-\infty}^{c} \phi\left(x \mid 2 c+\mu_{\mathrm{V}} \tau, \sigma_{\mathrm{V}}^{2} \tau\right) \Phi\left(x \mid c-\mu_{\mathrm{AV}} d^{\prime}, \sigma_{\mathrm{AV}}^{2} d^{\prime}\right) d x \\
& \quad-\exp \left(\frac{2 c \mu_{\mathrm{V}}}{\sigma_{\mathrm{V}}^{2}}+\frac{2 c \mu_{\mathrm{AV}}}{\sigma_{\mathrm{AV}}^{2}}\right) \int_{-\infty}^{c} \exp \left(-\frac{2 \mu_{\mathrm{AV}}}{\sigma_{\mathrm{AV}}^{2}} x\right) \phi\left(x \mid 2 c+\mu_{\mathrm{V}} \tau, \sigma_{\mathrm{V}}^{2} \tau\right) \Phi\left(x \mid c+\mu_{\mathrm{AV}} d^{\prime}, \sigma_{\mathrm{AV}}^{2} d^{\prime}\right)
\end{aligned}
$$

By completing the square, we have $\exp (r x) \phi\left(x \mid m_{1}, s_{1}^{2}\right)=\exp \left(r m_{1}+\frac{r^{2} s_{1}^{2}}{2}\right) \phi\left(x \mid m_{1}^{\prime}, s_{1}^{2}\right)$, with $m_{1}^{\prime}=m_{1}+r s_{1}^{2}$. Let $u=\frac{x-m_{1}^{\prime}}{s_{1}}$, or $x=u s_{1}+m_{1}^{\prime}$. Then, the integral can be rewritten as 


$$
\begin{aligned}
\int_{-\infty}^{c} \phi\left(x \mid m_{1}^{\prime}, s_{1}^{2}\right) \cdot \Phi\left(x \mid m_{2}, s_{2}^{2}\right) d x=\int_{-\infty}^{\left(c-m_{1}^{\prime}\right) / s_{1}} \phi(u) \cdot \Phi\left(u \mid \frac{m_{2}-m_{1}^{\prime}}{s_{1}}, \frac{s_{2}^{2}}{s_{1}^{2}}\right) d u \\
=\int_{-\infty}^{\left(c-m_{1}^{\prime}\right) / s_{1}} \phi(u) \cdot \Phi\left(\frac{s_{1}}{s_{2}} u+\frac{m_{1}^{\prime}-m_{2}}{s_{2}}\right) d u .
\end{aligned}
$$

549

550 The form of (A.4) now matches Owen (1980, Eq. 10,010.1) which can be determined by the bivariate

551 Normal distribution, $\int_{-\infty}^{y} \phi(u) \cdot \Phi(a+b u) d u=\Phi \Phi\left(\frac{a}{\sqrt{1+b^{2}}}, y \mid \varrho=-\frac{b}{\sqrt{1+b^{2}}}\right)$. An implementation in R (R 552 Core Team, 2017) is available as online supplementary material.

\section{Appendix B: Conditional mean response time}

554 Here we derive the explicit expressions for the conditional mean response time for bimodal stimuli with 555 onset asynchrony $\tau$, conditional on absorption before the deadline (Eqs. 13-15). We consider again the 556 case $\mathrm{V}(\tau) \mathrm{A}$ in which the visual stimulus is presented first. Between $t=0$ and $t=\tau$, only the visual channel contributes to the build-up of evidence, so the conditional mean RT is given by Schwarz (1994, Equation A.2).

559

560

561

$$
\begin{aligned}
& P\left(\mathbf{D}_{\mathrm{V}(\tau) \mathrm{A}} \leq \tau\right) \cdot E\left(\mathbf{D}_{\mathrm{V}(\tau) \mathrm{A}} \mid \mathbf{D}_{\mathrm{V}(\tau) \mathrm{A}} \leq \tau\right) \\
& \quad=\int_{0}^{\tau} t \cdot g\left(t \mid c, \mu_{\mathrm{V}}, \sigma_{\mathrm{V}}^{2}\right) d t=\frac{c}{\mu_{\mathrm{V}}}\left[\Phi\left(\mu_{\mathrm{V}} \tau \mid c, \sigma_{\mathrm{V}}^{2} \tau\right)-\exp \left(\frac{2 c \mu_{\mathrm{V}}}{\sigma_{\mathrm{V}}^{2}}\right) \Phi\left(-\mu_{\mathrm{V}} \tau \mid c, \sigma_{\mathrm{V}}^{2} \tau\right)\right]
\end{aligned}
$$

562

563

Later, within the time interval $(\tau, d)$, the expected detection time is again a mixture of expected detection 564 times for the processes still active at time $\tau$, weighted by the density of processes at $\mathbf{X}(\tau)$. These processes

565 now have increased drift $\mu_{\mathrm{AV}}$ and variance $\sigma_{\mathrm{AV}}^{2}$; the work to be done (i.e., the barrier) depends on the 566 activation $\mathbf{X}(\tau)<c$. Note that $\tau$ milliseconds have already passed since stimulus onset, hence the

567 remaining time is $d^{\prime}=d-\tau$.

568

569

570

571

572

$$
\begin{aligned}
P(\tau< & \left.\mathbf{D}_{\mathrm{V}(\tau) \mathrm{A}} \leq d\right) \cdot E\left(\mathbf{D}_{\mathrm{V}(\tau) \mathrm{A}} \mid \tau<\mathbf{D}_{\mathrm{V}(\tau) \mathrm{A}} \leq d\right) \\
= & \int_{-\infty}^{c} P[\mathbf{X}(\tau)=x] \cdot P\left(\mathbf{D}_{\mathrm{AV}} \leq d^{\prime} \mid \mathbf{X}(\tau)=x\right) \cdot\left\{\tau+E\left[\mathbf{D}_{\mathrm{AV}} \mid \mathbf{X}(\tau)=x, \mathbf{D}_{\mathrm{AV}} \leq d^{\prime}\right]\right\} d x \\
= & \int_{-\infty}^{c} w\left(x, \tau \mid c, \mu_{\mathrm{V}}, \sigma_{\mathrm{V}}^{2}\right) \cdot G\left(d^{\prime} \mid c-x, \mu_{\mathrm{AV}}, \sigma_{\mathrm{AV}}^{2}\right) d x \cdot \tau \\
& \quad+\int_{-\infty}^{c} w\left(x, \tau \mid c, \mu_{\mathrm{V}}, \sigma_{\mathrm{V}}^{2}\right) \cdot \int_{0}^{d^{\prime}} t \cdot g\left(t \mid c-x, \mu_{\mathrm{AV}}, \sigma_{\mathrm{AV}}^{2}\right) d t d x
\end{aligned}
$$


574 The first term corresponds exactly to (A.2), multiplied by the onset asynchrony $\tau$. See again Schwarz (1994,

575 Equation A.2) for $\int_{0}^{d^{\prime}} t \cdot g\left(t \mid c-x, \mu_{\mathrm{AV}}, \sigma_{\mathrm{AV}}^{2}\right) d t$. The double integral in (B.2) can then be rewritten as

576

577

$\int_{-\infty}^{c}\left[\phi\left(x \mid \mu_{\mathrm{V}} \tau, \sigma_{\mathrm{V}}^{2} \tau\right)-\exp \left(\frac{2 c \mu_{\mathrm{V}}}{\sigma_{\mathrm{V}}^{2}}\right) \cdot \phi\left(x \mid 2 c+\mu_{\mathrm{V}} \tau, \sigma_{\mathrm{V}}^{2} \tau\right)\right]$

578

$\times \frac{c-x}{\mu_{\mathrm{AV}}} \cdot\left[\Phi\left(x \mid c-\mu_{\mathrm{AV}} d^{\prime}, \sigma_{\mathrm{AV}}^{2} d^{\prime}\right)-\exp \left(\frac{2(c-x) \mu_{\mathrm{AV}}}{\sigma_{\mathrm{AV}}^{2}}\right) \Phi\left(x \mid c+\mu_{\mathrm{AV}} d^{\prime}, \sigma_{\mathrm{AV}}^{2} d^{\prime}\right)\right] d x$

579

$$
=\frac{c}{\mu_{\mathrm{AV}}} \int_{-\infty}^{c} \phi\left(x \mid \mu_{\mathrm{V}} \tau, \sigma_{\mathrm{V}}^{2} \tau\right) \Phi\left(x \mid c-\mu_{\mathrm{AV}} d^{\prime}, \sigma_{\mathrm{AV}}^{2} d^{\prime}\right) d x
$$

580

581

$$
-\frac{c}{\mu_{\mathrm{AV}}} \exp \left(\frac{2 c \mu_{\mathrm{V}}}{\sigma_{\mathrm{V}}^{2}}\right) \int_{-\infty}^{c} \phi\left(x \mid 2 c+\mu_{\mathrm{V}} \tau, \sigma_{\mathrm{V}}^{2} \tau\right) \Phi\left(x \mid c-\mu_{\mathrm{AV}} d^{\prime}, \sigma_{\mathrm{AV}}^{2} d^{\prime}\right) d x
$$$$
-\frac{c}{\mu_{\mathrm{AV}}} \exp \left(\frac{2 c \mu_{\mathrm{AV}}}{\sigma_{\mathrm{AV}}^{2}}\right) \int_{-\infty}^{c} \exp \left(-\frac{2 \mu_{\mathrm{AV}} x}{\sigma_{\mathrm{AV}}^{2}}\right) \phi\left(x \mid \mu_{\mathrm{V}} \tau, \sigma_{\mathrm{V}}^{2} \tau\right) \Phi\left(x \mid c+\mu_{\mathrm{AV}} d^{\prime}, \sigma_{\mathrm{AV}}^{2} d^{\prime}\right) d x
$$

582

$$
+\frac{c}{\mu_{\mathrm{AV}}} \exp \left(\frac{2 c \mu_{\mathrm{V}}}{\sigma_{\mathrm{V}}^{2}}+\frac{2 c \mu_{\mathrm{AV}}}{\sigma_{\mathrm{AV}}^{2}}\right) \int_{-\infty}^{c} \exp \left(-\frac{2 \mu_{\mathrm{AV}} x}{\sigma_{\mathrm{AV}}^{2}}\right) \phi\left(x \mid 2 c+\mu_{\mathrm{V}} \tau, \sigma_{\mathrm{V}}^{2} \tau\right) \Phi\left(x \mid c+\mu_{\mathrm{AV}} d^{\prime}, \sigma_{\mathrm{AV}}^{2} d^{\prime}\right) d x
$$

583

$$
-\frac{1}{\mu_{\mathrm{AV}}} \int_{-\infty}^{c} x \phi\left(x \mid \mu_{\mathrm{V}} \tau, \sigma_{\mathrm{V}}^{2} \tau\right) \Phi\left(x \mid c-\mu_{\mathrm{AV}} d^{\prime}, \sigma_{\mathrm{AV}}^{2} d^{\prime}\right) d x
$$

584

585

$$
+\frac{1}{\mu_{\mathrm{AV}}} \exp \left(\frac{2 c \mu_{\mathrm{V}}}{\sigma_{\mathrm{V}}^{2}}\right) \int_{-\infty}^{c} x \phi\left(x \mid 2 c+\mu_{\mathrm{V}} \tau, \sigma_{\mathrm{V}}^{2} \tau\right) \Phi\left(x \mid c-\mu_{\mathrm{AV}} d^{\prime}, \sigma_{\mathrm{AV}}^{2} d^{\prime}\right) d x
$$

586

$$
+\frac{1}{\mu_{\mathrm{AV}}} \exp \left(\frac{2 c \mu_{\mathrm{AV}}}{\sigma_{\mathrm{AV}}^{2}}\right) \int_{-\infty}^{c} x \exp \left(-\frac{2 \mu_{\mathrm{AV}} x}{\sigma_{\mathrm{AV}}^{2}}\right) \phi\left(x \mid \mu_{\mathrm{V}} \tau, \sigma_{\mathrm{V}}^{2} \tau\right) \Phi\left(x \mid c+\mu_{\mathrm{AV}} d^{\prime}, \sigma_{\mathrm{AV}}^{2} d^{\prime}\right) d x
$$

$$
-\frac{1}{\mu_{\mathrm{AV}}} \exp \left(\frac{2 c \mu_{\mathrm{V}}}{\sigma_{\mathrm{V}}^{2}}+\frac{2 c \mu_{\mathrm{AV}}}{\sigma_{\mathrm{AV}}^{2}}\right) \int_{-\infty}^{c} x \exp \left(-\frac{2 \mu_{\mathrm{AV}} x}{\sigma_{\mathrm{AV}}^{2}}\right) \phi\left(x \mid 2 c+\mu_{\mathrm{V}} \tau, \sigma_{\mathrm{V}}^{2} \tau\right) \Phi\left(x \mid c+\mu_{\mathrm{AV}} d^{\prime}, \sigma_{\mathrm{AV}}^{2} d^{\prime}\right) d x
$$

587

588 The first four terms correspond to (A.3), multiplied by a constant ( $\left.\pm c / \mu_{\mathrm{AV}}\right)$. By completing the square, we

589 have again $\exp (r x) \phi\left(x \mid m_{1}, s_{1}^{2}\right)=\exp \left(r m_{1}+\frac{r^{2} s_{1}^{2}}{2}\right) \phi\left(x \mid m_{1}^{\prime}, s_{1}^{2}\right)$, with $m_{1}^{\prime}=m_{1}+r s_{1}^{2}$.

590 Let $u=\frac{x-m_{1}^{\prime}}{s_{1}}$, or $x=m_{1}^{\prime}+u s_{1}$. Then, the integral can be rewritten as

591

592

593

$$
\begin{aligned}
\int_{-\infty}^{c} x & \cdot \phi\left(x \mid m_{1}^{\prime}, s_{1}^{2}\right) \cdot \Phi\left(x \mid m_{2}, s_{2}^{2}\right) d x \\
& =\int_{-\infty}^{\left(c-m_{1}^{\prime}\right) / s_{1}}\left(m_{1}^{\prime}+u s_{1}\right) \cdot \phi(u \mid 0,1) \cdot \Phi\left(u \mid \frac{m_{2}-m_{1}^{\prime}}{s_{1}}, \frac{s_{2}^{2}}{s_{1}^{2}}\right) d u
\end{aligned}
$$




$$
=m_{1}^{\prime} \cdot \int_{-\infty}^{\left(c-m_{1}^{\prime}\right) / s_{1}} \phi(u \mid 0,1) \cdot \Phi\left(\frac{s_{1}}{s_{2}} u+\frac{m_{1}^{\prime}-m_{2}}{s_{2}} \mid 0,1\right) d u
$$

595

$$
+s_{1} \cdot \int_{-\infty}^{\left(c-m_{1}^{\prime}\right) / s_{1}} u \cdot \phi(u \mid 0,1) \cdot \Phi\left(\frac{s_{1}}{s_{2}} u+\frac{m_{1}^{\prime}-m_{2}}{s_{2}} \mid 0,1\right) d u
$$

596

597 The first term of (B.4) matches again (Owen, 1980, Eq. 10,010.1). The second term matches Eq. 10,010.1 598 from (Owen, 1980, Eq. 10,010.1) and is calculated by

$599 \quad \int_{-\infty}^{y} u \cdot \phi(u) \cdot \Phi(a+b u) d u=\frac{b}{\sqrt{1+b^{2}}} \phi\left(\frac{b}{\sqrt{1+b^{2}}}\right) \Phi\left(y \sqrt{1+b^{2}}+\frac{a b}{\sqrt{1+b^{2}}}\right)-\phi(y) \Phi(a+b u)$. A

600 implementation in R (R Core Team, 2017) is available as an online supplement. 
bioRxiv preprint doi: https://doi.org/10.1101/173773; this version posted September 5, 2017. The copyright holder for this preprint (which was not certified by peer review) is the author/funder, who has granted bioRxiv a license to display the preprint in perpetuity. It is made available under aCC-BY-NC-ND 4.0 International license.

\section{Author Contributions}

CC and MG designed the experiment. CC performed experiments. CC and MG analyzed the data. MG developed the formulation of the deadline model. CC and MG wrote the paper. Both authors evaluated data and modeling results. 


\section{Accuracy and mean response time in a diffusion superposition model with deadline}

Online supplement for "Audiovisual detection at different intensities and delays"

This online supplement provides implementation details on the derivation of the predictions for mean response time and accuracy for the diffusion superposition model (Diederich, 1995; Schwarz, 1994) with a deadline. For simplicity, we reiterate the relevant parts of the methods section here and then add code in $R$ statistical language for the different equations. The R code (R Core Team, 2017) includes the necessary defaults that allow testing and deployment in other analyses.

\section{Libraries}

The code requires the inverse Gaussian distribution package SuppDists (Wheeler, 2013, available from CRAN). In addition, package mvtnorm (Genz et al., 2014) is used for the bivariate Normal distribution.

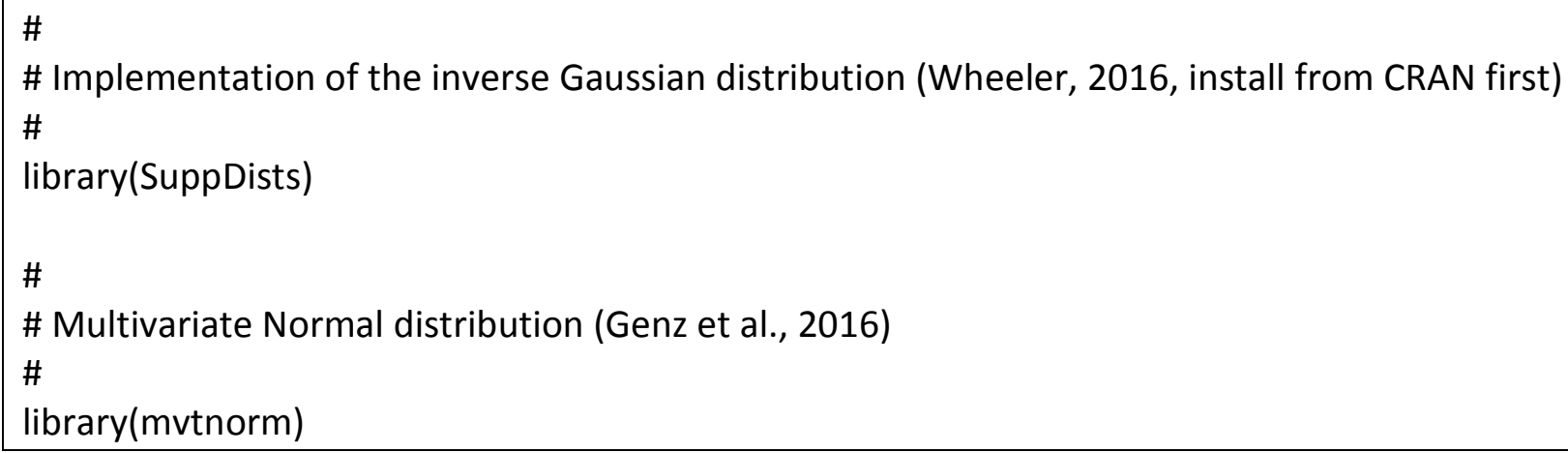

\section{Accuracy}

For unimodal/synchronous stimuli, accuracy is given by the inverse Gaussian distribution at the deadline $d$. For example, Monkey 1's accuracy in Condition v (low intensity) is given by acc_sync(d=1000, c=100, mu=0.13, sigma $\left.2=4.3^{\wedge} 2\right)$.

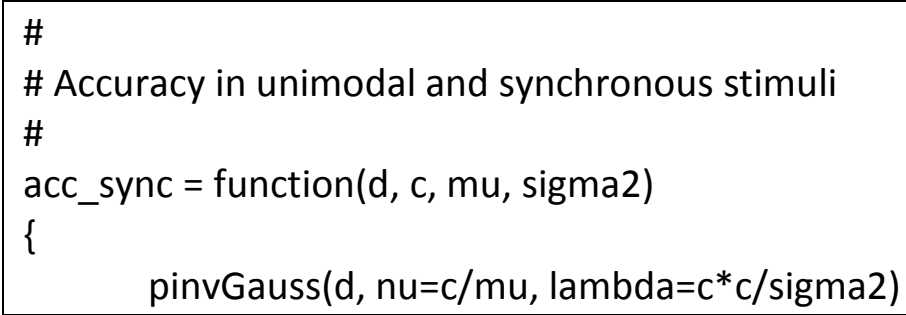


For stimuli with onset asynchrony $\tau$, accuracy is given by (12) which is the sum of the inverse Gaussian distribution at time $\tau$ and four integrals of the form $q \cdot \int_{-\infty}^{c} \exp (r x) \cdot \phi\left(x \mid m_{1}, s_{1}^{2}\right) \cdot \Phi\left(x \mid m_{2}, s_{2}^{2}\right) d x$ :

1. $q=1, r=0, m_{1}=\mu_{\mathrm{V}} \tau, s_{1}^{2}=\sigma_{\mathrm{V}}^{2} \tau, m_{2}=c-\mu_{\mathrm{AV}} d^{\prime}, s_{2}^{2}=\sigma_{\mathrm{AV}}^{2} d^{\prime}$.

2. $q=\exp \left(\frac{2 c \mu_{\mathrm{AV}}}{\sigma_{\mathrm{AV}}^{2}}\right), r=-\frac{2 \mu_{\mathrm{AV}}}{\sigma_{\mathrm{AV}}^{2}}, m_{1}=\mu_{\mathrm{V}} \tau, s_{1}^{2}=\sigma_{\mathrm{V}}^{2} \tau, m_{2}=c+\mu_{\mathrm{AV}} d^{\prime}, s_{2}^{2}=\sigma_{\mathrm{AV}}^{2} d^{\prime}$.

3. $q=-\exp \left(\frac{2 c \mu_{\mathrm{V}}}{\sigma_{\mathrm{V}}^{2}}\right), r=0, m_{1}=2 c+\mu_{\mathrm{V}} \tau, s_{1}^{2}=\sigma_{\mathrm{V}}^{2} \tau, m_{2}=c-\mu_{\mathrm{AV}} d^{\prime}, s_{2}^{2}=\sigma_{\mathrm{AV}}^{2} d^{\prime}$

4. $q=-\exp \left(\frac{2 c \mu_{\mathrm{V}}}{\sigma_{\mathrm{V}}^{2}}+\frac{2 c \mu_{\mathrm{AV}}}{\sigma_{\mathrm{AV}}^{2}}\right), r=-\frac{2 \mu_{\mathrm{AV}}}{\sigma_{\mathrm{AV}}^{2}}, m_{1}=2 c+\mu_{\mathrm{V}} \tau, s_{1}^{2}=\sigma_{\mathrm{V}}^{2} \tau, m_{2}=c+\mu_{\mathrm{AV}} d^{\prime}, s_{2}^{2}=\sigma_{\mathrm{AV}}^{2} d^{\prime}$

with $d^{\prime}=d-\tau$. Three convenience functions evaluate these integrals using Eq. 10,010.1 in (Owen, 1980):

$$
\begin{aligned}
& \int_{-\infty}^{c} \exp (r x) \phi\left(x \mid m_{1}, s_{1}^{2}\right) \Phi\left(x \mid m_{2}, s_{2}^{2}\right) d x=\exp \left(r m_{1}+\frac{r^{2} s_{1}^{2}}{2}\right) \int_{-\infty}^{c} \phi\left(x \mid m_{1}^{\prime}, s_{1}^{2}\right) \cdot \Phi\left(x \mid m_{2}, s_{2}^{2}\right) d x, \\
& \int_{-\infty}^{c} \phi\left(x \mid m_{1}^{\prime}, s_{1}^{2}\right) \cdot \Phi\left(x \mid m_{2}, s_{2}^{2}\right) d x=\int_{-\infty}^{\left(c-m_{1}^{\prime}\right) / s_{1}} \phi(u) \cdot \Phi\left(\frac{s_{1}}{s_{2}} u+\frac{m_{1}^{\prime}-m_{2}}{s_{2}}\right) d u, \\
& \int_{-\infty}^{y} \phi(u) \cdot \Phi(a+b u) d u=\Phi \Phi\left(\frac{a}{\sqrt{1+b^{2}}}, y \mid \varrho=-\frac{b}{\sqrt{1+b^{2}}}\right), \text { with } m_{1}^{\prime}=m_{1}+r s_{1}^{2} .
\end{aligned}
$$

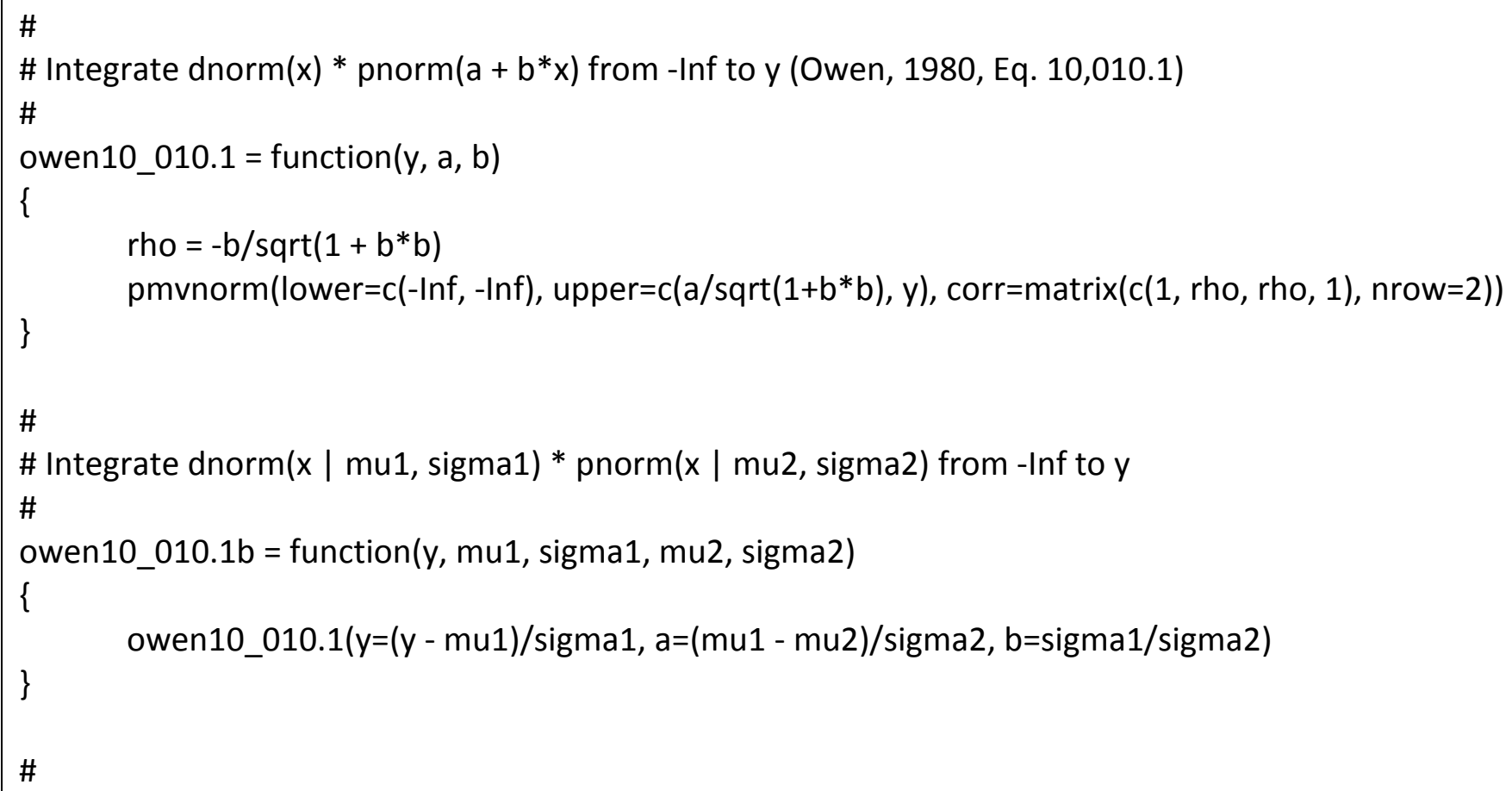




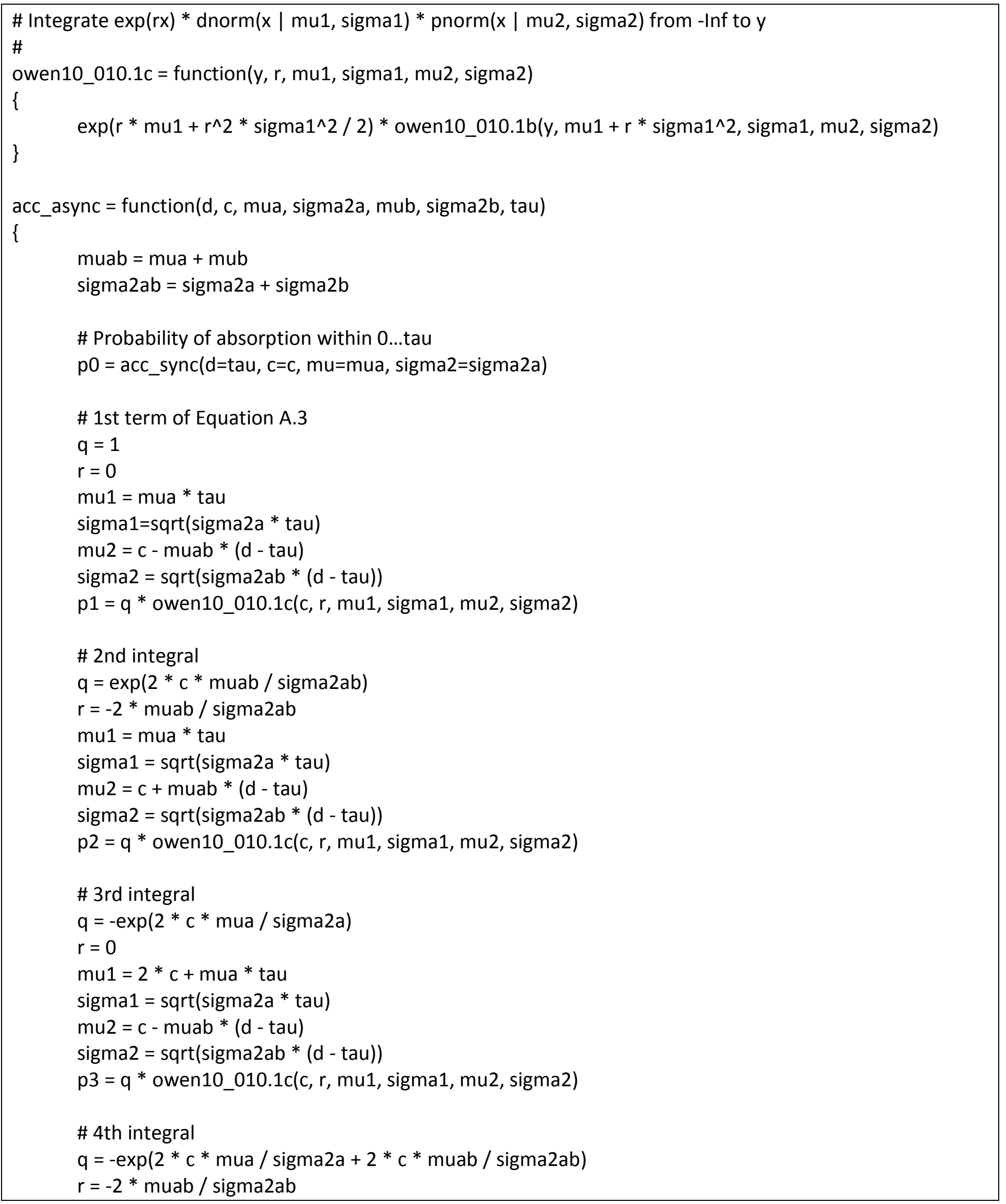




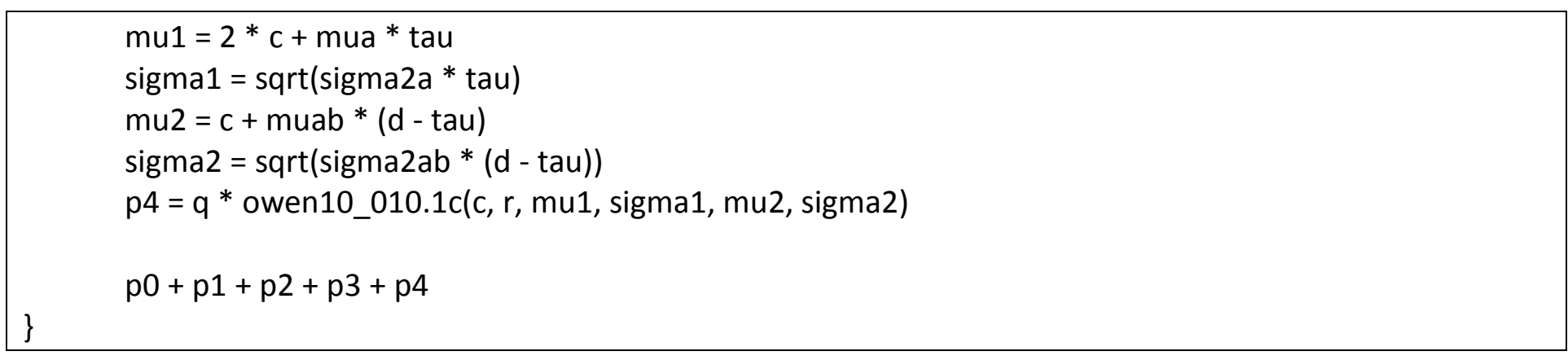

For example, Monkey 1's accuracy in Condition v100a (low intensity) is given by acc_async(d=1000, $c=100$, mua $=0.13$, sigma $2 a=4.3^{\wedge} 2$, mub=0.34, sigma $2 b=11.7^{\wedge} 2$, tau $\left.=67\right)$.

\section{Mean response time}

For unimodal/synchronous stimuli, the mean RT is given by Equation 4 which integrates the product of the time and the inverse Gaussian density from zero until the deadline $d$. For example, Monkey $1^{\prime}$ s mean RT in Condition v (low intensity) is given by mrt_sync(d=1000, $c=100, \mathrm{mu}=0.13$, sigma2 $\left.=4.3^{\wedge} 2\right)$.

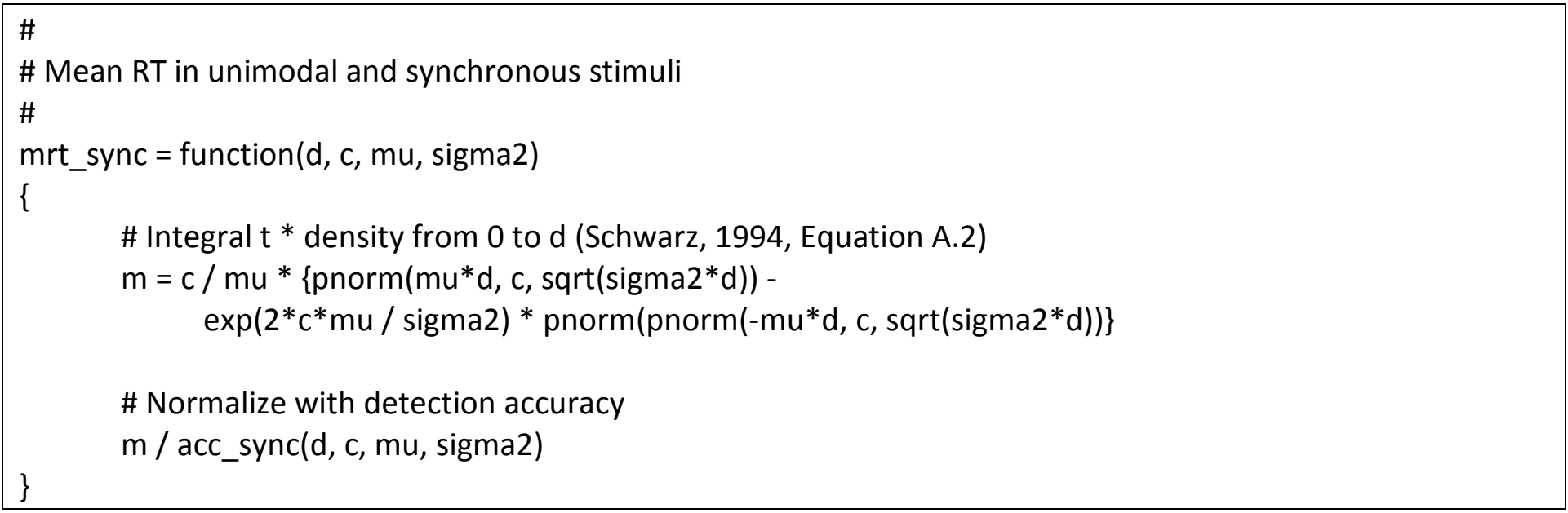

For stimuli with onset asynchrony $\tau$, mean RT is given by Equations 13-15 which boil down to Equations 4 and 12 , and four integrals of the form $q \cdot \int_{-\infty}^{c} \exp (r x) \cdot \phi\left(x \mid m_{1}, s_{1}^{2}\right) \cdot \Phi\left(x \mid m_{2}, s_{2}^{2}\right) d x$, as well as four integrals of the form $q \cdot \int_{-\infty}^{c} x \cdot \exp (r x) \cdot \phi\left(x \mid m_{1}, s_{1}^{2}\right) \cdot \Phi\left(x \mid m_{2}, s_{2}^{2}\right) d x$

1. $q=\frac{c}{\mu_{\mathrm{AV}}}, r=0, m_{1}=\mu_{\mathrm{V}} \tau, s_{1}^{2}=\sigma_{\mathrm{V}}^{2} \tau, m_{2}=c-\mu_{\mathrm{AV}} d^{\prime}, s_{2}^{2}=\sigma_{\mathrm{AV}}^{2} d^{\prime}$.

2. $q=-\frac{c}{\mu_{\mathrm{AV}}} \cdot \exp \left(-\frac{2 c \mu_{\mathrm{AV}}}{\sigma_{\mathrm{AV}}^{2}}\right), r=-\frac{2 \mu_{\mathrm{AV}}}{\sigma_{\mathrm{AV}}^{2}}, m_{1}=\mu_{\mathrm{V}} \tau, s_{1}^{2}=\sigma_{\mathrm{V}}^{2} \tau, m_{2}=c+\mu_{\mathrm{AV}} d^{\prime}, s_{2}^{2}=\sigma_{\mathrm{AV}}^{2} d^{\prime}$.

3. $q=-\frac{c}{\mu_{\mathrm{AV}}} \cdot \exp \left(\frac{2 c \mu_{\mathrm{V}}}{\sigma_{\mathrm{V}}^{2}}\right), r=0, m_{1}=2 c+\mu_{\mathrm{V}} \tau, s_{1}^{2}=\sigma_{\mathrm{V}}^{2} \tau, m_{2}=c-\mu_{\mathrm{AV}} d^{\prime}, s_{2}^{2}=\sigma_{\mathrm{AV}}^{2} d^{\prime}$ 

4. $q=\frac{c}{\mu_{\mathrm{AV}}} \cdot \exp \left(\frac{2 c \mu_{\mathrm{V}}}{\sigma_{\mathrm{V}}^{2}}+\frac{2 c \mu_{\mathrm{AV}}}{\sigma_{\mathrm{AV}}^{2}}\right), r=-\frac{2 \mu_{\mathrm{AV}}}{\sigma_{\mathrm{AV}}^{2}}, m_{1}=2 c+\mu_{\mathrm{V}} \tau, s_{1}^{2}=\sigma_{\mathrm{V}}^{2} \tau, m_{2}=c+\mu_{\mathrm{AV}} d^{\prime}, s_{2}^{2}=\sigma_{\mathrm{AV}}^{2} d^{\prime}$
5. $q=\frac{-1}{\mu_{\mathrm{AV}}}, r=0, m_{1}=\mu_{\mathrm{V}} \tau, s_{1}^{2}=\sigma_{\mathrm{V}}^{2} \tau, m_{2}=c-\mu_{\mathrm{AV}} d^{\prime}, s_{2}^{2}=\sigma_{\mathrm{AV}}^{2} d^{\prime}$.
6. $q=\frac{1}{\mu_{\mathrm{AV}}} \cdot \exp \left(\frac{2 c \mu_{\mathrm{V}}}{\sigma_{\mathrm{V}}^{2}}\right), r=0, m_{1}=2 c+\mu_{\mathrm{V}} \tau, s_{1}^{2}=\sigma_{\mathrm{V}}^{2} \tau, m_{2}=c-\mu_{\mathrm{AV}} d^{\prime}, s_{2}^{2}=\sigma_{\mathrm{AV}}^{2} d^{\prime}$
7. $q=\frac{1}{\mu_{\mathrm{AV}}} \cdot \exp \left(-\frac{2 c \mu_{\mathrm{AV}}}{\sigma_{\mathrm{AV}}^{2}}\right), r=-\frac{2 \mu_{\mathrm{AV}}}{\sigma_{\mathrm{AV}}^{2}}, m_{1}=\mu_{\mathrm{V}} \tau, s_{1}^{2}=\sigma_{\mathrm{V}}^{2} \tau, m_{2}=c+\mu_{\mathrm{AV}} d^{\prime}, s_{2}^{2}=\sigma_{\mathrm{AV}}^{2} d^{\prime}$.
8. $q=\frac{-1}{\mu_{\mathrm{AV}}} \cdot \exp \left(\frac{2 c \mu_{\mathrm{V}}}{\sigma_{\mathrm{V}}^{2}}+\frac{2 c \mu_{\mathrm{AV}}}{\sigma_{\mathrm{AV}}^{2}}\right), r=-\frac{2 \mu_{\mathrm{AV}}}{\sigma_{\mathrm{AV}}^{2}}, m_{1}=2 c+\mu_{\mathrm{V}} \tau, s_{1}^{2}=\sigma_{\mathrm{V}}^{2} \tau, m_{2}=c+\mu_{\mathrm{AV}} d^{\prime}, s_{2}^{2}=\sigma_{\mathrm{AV}}^{2} d^{\prime}$

We defined again convenience functions that transform these integrals to two expressions that match

Equations 10,010.1 and 10,011.1 in (Owen, 1980):

$$
\begin{aligned}
& \int_{-\infty}^{c} x \exp (r x) \phi\left(x \mid m_{1}, s_{1}^{2}\right) \Phi\left(x \mid m_{2}, s_{2}^{2}\right) d x \\
& =\exp \left(r m_{1}+\frac{r^{2} s_{1}^{2}}{2}\right) \int_{-\infty}^{c} x \cdot \phi\left(x \mid m_{1}^{\prime}, s_{1}^{2}\right) \cdot \Phi\left(x \mid m_{2}, s_{2}^{2}\right) d x, \\
& \int_{-\infty}^{c} x \cdot \phi\left(x \mid m_{1}^{\prime}, s_{1}^{2}\right) \cdot \Phi\left(x \mid m_{2}, s_{2}^{2}\right) d x \\
& =\int_{-\infty}^{\frac{\left(c-m_{1}^{\prime}\right)}{s_{1}}}\left(s_{1} u+m_{1}^{\prime}\right) \cdot \phi(u) \cdot \Phi\left(\frac{s_{1}}{s_{2}} u+\frac{m_{1}^{\prime}-m_{2}}{s_{2}}\right) d u \\
& =m_{1}^{\prime} \int_{-\infty}^{\frac{c-m_{1}^{\prime}}{s_{1}}} \phi(u) \cdot \Phi\left(\frac{s_{1}}{s_{2}} u+\frac{m_{1}^{\prime}-m_{2}}{s_{2}}\right) d u \\
& +s_{1} \int_{-\infty}^{\frac{c-m_{1}^{\prime}}{s_{1}}} u \cdot \phi(u) \cdot \Phi\left(\frac{s_{1}}{s_{2}} u+\frac{m_{1}^{\prime}-m_{2}}{s_{2}}\right) d u \\
& \int_{-\infty}^{y} \phi(u) \cdot \Phi(a+b u) d u=(\text { see above) } \\
& \int_{-\infty}^{y} u \cdot \phi(u) \cdot \Phi(a+b u) d u=\frac{b}{\sqrt{1+b^{2}}} \cdot \phi\left(\frac{a}{\sqrt{1+b^{2}}}\right) \cdot \Phi\left(u \sqrt{1+b^{2}}+\frac{a b}{\sqrt{1+b^{2}}}\right)-\phi(u) \cdot \Phi(a+b u)
\end{aligned}
$$

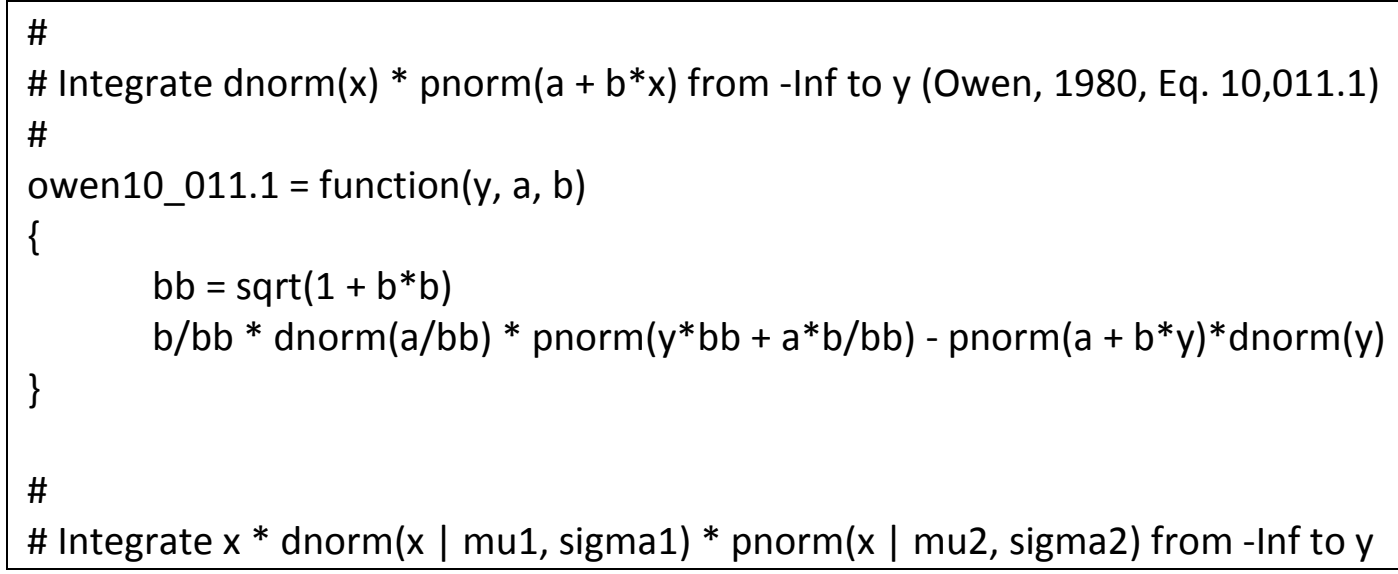




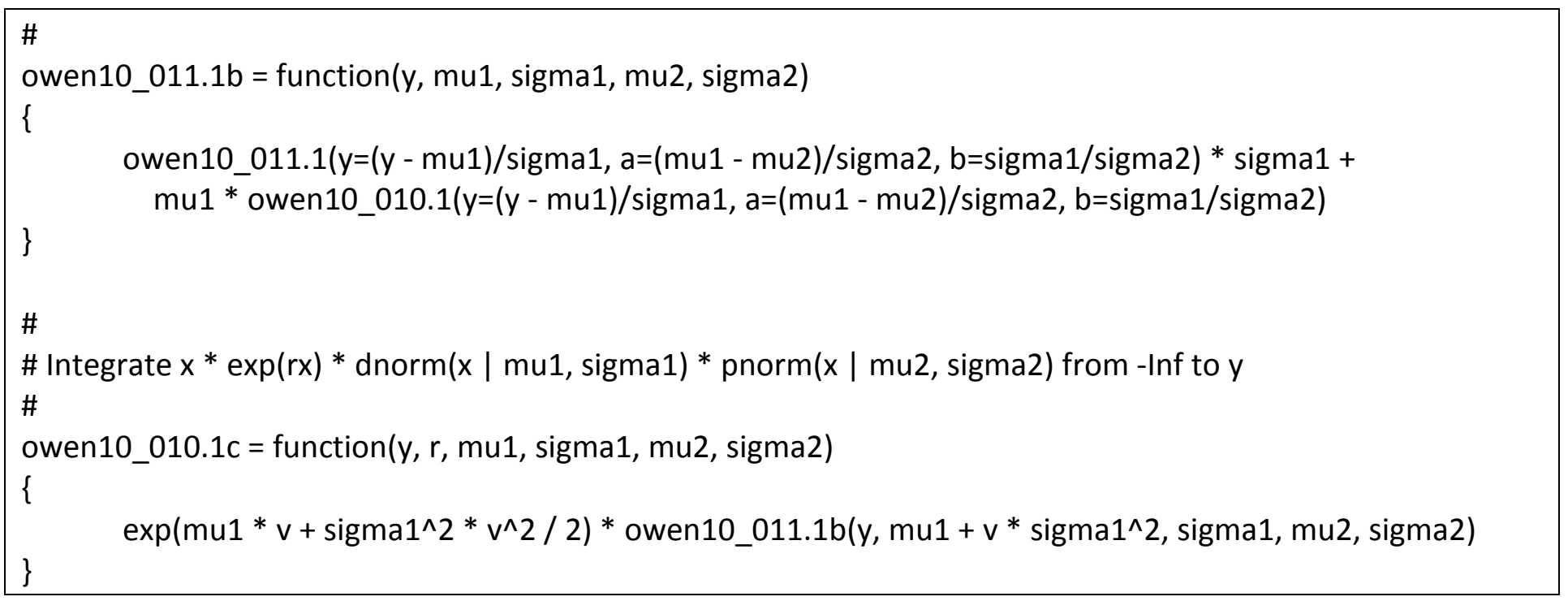

For example, Monkey 1's mean RT in Condition v100a (low intensity) is predicted to mrt_async(d=1000, c=100, mua=0.13, sigma2a $=4.3^{\wedge} 2$, mub=0.34, sigma $2 b=11.7^{\wedge} 2$, tau $\left.=67\right)$.

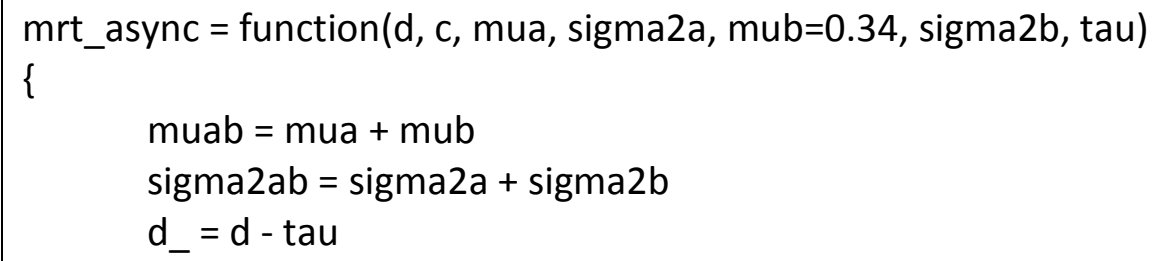


mu1 $=$ mua $*$ tau

sigma1 $=\operatorname{sqrt}(\operatorname{sigma} 2 \mathrm{a} *$ tau $)$

mu2 $=\mathrm{c}+$ muab $* \mathrm{~d}_{-}$

sigma2 $=\operatorname{sqrt}\left(\operatorname{sigma} 2 a b * d_{\_}\right)$

$\mathrm{m} 2$ = $\mathrm{q}$ * owen10_010.1c(c, r, mu1, sigma1, mu2, sigma2)

\# 3rd integral

$q=-c /$ muab $* \exp (2 * c *$ mua / sigma2a $)$

$r=0$

mu1 $=2 * c+$ mua $*$ tau

sigma $1=\operatorname{sqrt}(\operatorname{sigma} 2 \mathrm{a} *$ tau $)$

mu2 $=\mathrm{c}-$ muab $* \mathrm{~d}_{-}$

$\operatorname{sigma2}=\operatorname{sqrt}\left(\operatorname{sigma} 2 a b * d_{-}\right)$

m3 = q * owen10_010.1c(c, r, mu1, sigma1, mu2, sigma2)

\# 4th integral

$\mathrm{q}=\mathrm{c} /$ muab $* \exp (2 * \mathrm{c} *$ mua $/ \operatorname{sigma} 2 \mathrm{a}+2 * \mathrm{c} *$ muab / sigma2ab $)$

$r=-2 *$ muab / sigma $2 a b$

$\operatorname{mu} 1=2 * c+$ mua $*$ tau

sigma $1=\operatorname{sqrt}(\operatorname{sigma} 2 \mathrm{a} *$ tau $)$

mu2 $=\mathrm{c}+\operatorname{muab}^{*} \mathrm{~d}_{-}$

$\operatorname{sigma2}=\operatorname{sqrt}\left(\operatorname{sigma} 2 \mathrm{ab} * \mathrm{~d}_{-}\right)$

m4 = q * owen10_010.1c(c, r, mu1, sigma1, mu2, sigma2)

\# 5th integral in B.3

$q=-1 /$ mua

$r=0$

mu1 $=$ mua $*$ tau

sigma1 $=\operatorname{sqrt}(\operatorname{sigma} 2 \mathrm{a} *$ tau $)$

mu2 $=\mathrm{c}-\mathrm{muab}^{*} \mathrm{~d}_{-}$

$\operatorname{sigma2}=\operatorname{sqrt}\left(\operatorname{sigma} 2 a b * d_{-}\right)$

m5 = q * owen10_011.1c(c, r, mu1, sigma1, mu2, sigma2)

\# 6th integral

$q=1 /$ muab * exp $(2 * c *$ muab / sigma $2 a b)$

$r=-2 *$ muab / sigma $2 a b$

mu1 = mua $*$ tau

sigma $1=\operatorname{sqrt}(\operatorname{sigma} 2 \mathrm{a} *$ tau $)$

mu2 $=\mathrm{c}+$ muab $^{*} \mathrm{~d}_{-}$

$\operatorname{sigma2}=\operatorname{sqrt}\left(\operatorname{sigma} 2 a b * d_{\_}\right)$

$\mathrm{m} 6=\mathrm{q}$ * owen10_011.1c(c, r, mu1, sigma1, mu2, sigma2)

\# 7th integral

$q=1 /$ muab $* \exp (2 * c *$ mua / sigma $2 a)$

$r=0$ 


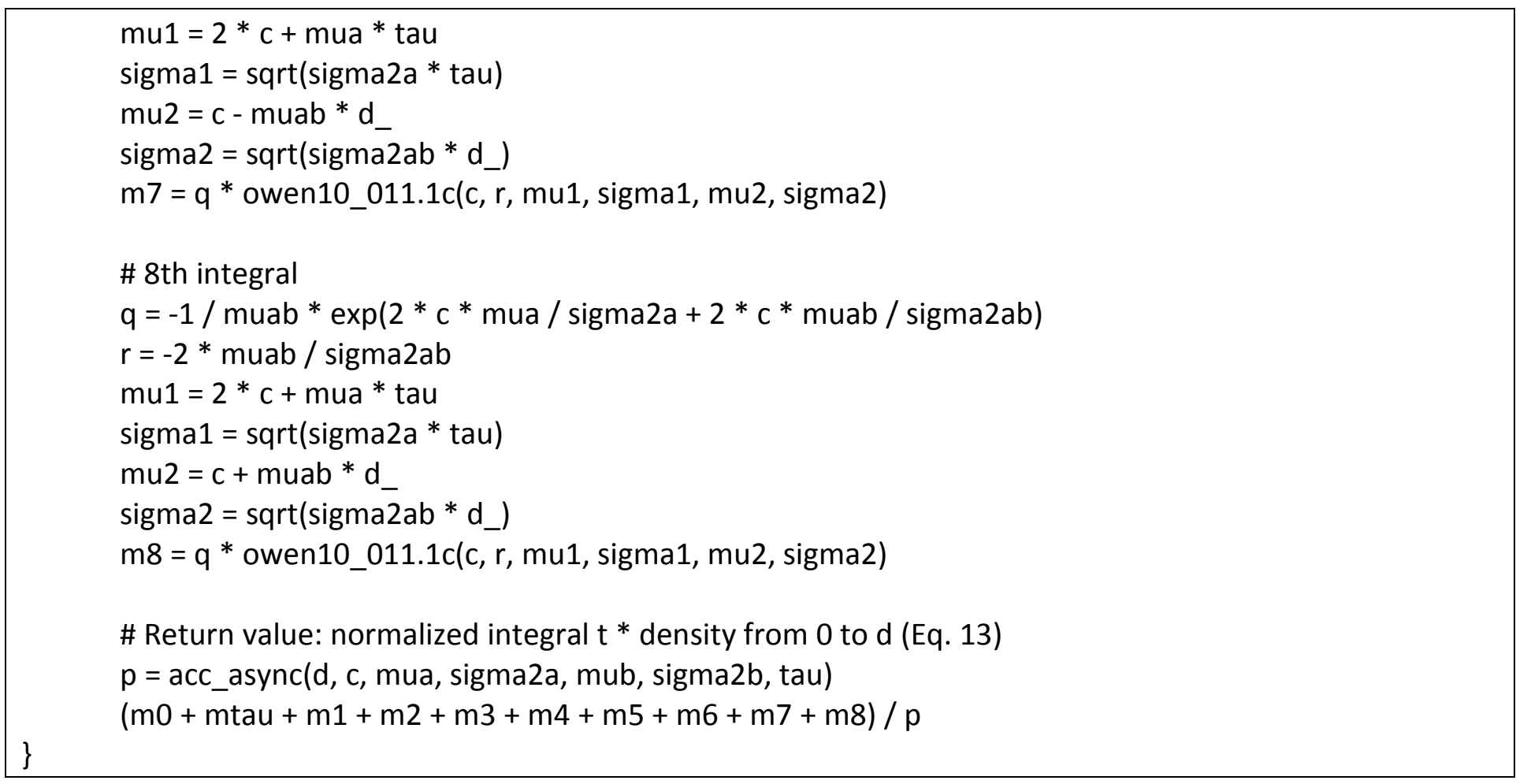

\section{References}

Beck, J. M., Ma, W. J., Kiani, R., Hanks, T., Churchland, A. K., Roitman, J., et al. (2008). Probabilistic population codes for Bayesian decision making. Neuron, 60(6), 1142-1152.

Besle, J., Fort, A., Delpuech, C., \& Giard, M.-H. (2004). Bimodal speech: early suppressive visual effects in human auditory cortex. Eur J Neurosci, 20(8), 2225-2234.

Chandrasekaran, C. (2016). Computational Models and Principles of Multisensory Integration. Current Opinion in Neurobiology, 43(Neurobiology of Learning and Plasticity).

Chandrasekaran, C., Lemus, L., \& Ghazanfar, A. A. (2013). Dynamic faces speed up the onset of auditory cortical spiking responses during vocal detection. Proc Natl Acad Sci U S A, 110(48), E4668-4677.

Chandrasekaran, C., Lemus, L., Trubanova, A., Gondan, M., \& Ghazanfar, A. (2011). Monkeys and Humans Share a Common Computation for Face/Voice Integration. PLoS Comput Biol, 7(9), e1002165.

Chandrasekaran, C., Turesson, H. K., Brown, C. H., \& Ghazanfar, A. A. (2010). The Influence of Natural Scene Dynamics on Auditory Cortical Activity. J. Neurosci., 30(42), 13919-13931.

Cluff, T., Crevecoeur, F., \& Scott, S. H. (2015). A perspective on multisensory integration and rapid perturbation responses. Vision Res, 110(Pt B), 215-222.

Colonius, H., \& Diederich, A. (2006). The race model inequality: interpreting a geometric measure of the amount of violation. Psychol Rev, 113(1), 148-154.

Cox, D. R., \& Miller, H. D. (1965). The Theory of Stochastic Processes. New York: Wiley.

Crevecoeur, F., Munoz, D. P., \& Scott, S. H. (2016). Dynamic Multisensory Integration: Somatosensory Speed Trumps Visual Accuracy during Feedback Control. J Neurosci, 36(33), 8598-8611.

Dahl, C. D., Logothetis, N. K., \& Kayser, C. (2010). Modulation of visual responses in the superior temporal sulcus by audio-visual congruency. Front Integr Neurosci, 4, 10. 
Diederich, A. (1995). Intersensory Facilitation of Reaction Time: Evaluation of Counter and Diffusion Coactivation Models. Journal of Mathematical Psychology, 39(2), 197-215.

Diederich, A., \& Colonius, H. (1987). Intersensory facilitation in the motor component? Psychological Research, 49(1), 23-29.

Diederich, A., \& Colonius, H. (2004a). Bimodal and trimodal multisensory enhancement: effects of stimulus onset and intensity on reaction time. Percept Psychophys, 66(8), 1388-1404.

Diederich, A., \& Colonius, H. (2004b). Modeling the time course of multimodal interaction in manual and saccadic responses. In G. Calvert, C. Spence \& B. Stein (Eds.), Handbook of Multisensory Processes (pp. 395 408). Cambridge, MA: MIT Press,.

Dixon, N. F., \& Spitz, L. (1980). The Detection of Auditory Visual Desynchrony. Perception, 719-721.

Drugowitsch, J., DeAngelis, G. C., Klier, E. M., Angelaki, D. E., \& Pouget, A. (2014). Optimal multisensory decision-making in a reaction-time task. Elife (Cambridge), e03005.

Egan, J. P., Greenberg, G. Z., \& Schulman, A. I. (1961). Operating Characteristics, Signal Detectability, and the Method of Free Response. The Journal of the Acoustical Society of America, 33(8), 993-1007.

Eriksen, C. W. (1988). A source of error in attempts to distinguish coactivation from separate activation in the perception of redundant targets. Percept Psychophys, 44(2), 191-193.

Fetsch, C. R., DeAngelis, G. C., \& Angelaki, D. E. (2013). Bridging the gap between theories of sensory cue integration and the physiology of multisensory neurons. Nat Rev Neurosci, 14(6), 429-442.

Flanagan, J. L., \& Golden, R. M. (1966). Phase Vocoder. Bell System Technical Journal, 1493-1509.

Gondan, M., Götze, C., \& Greenlee, M. W. (2010). Redundancy gains in simple responses and go/no-go tasks. Atten Percept Psychophys, 72(6), 1692-1709.

Gondan, M., \& Heckel, A. (2008). Testing the race inequality: A simple correction procedure for fast guesses. Journal of Mathematical Psychology, 52(5), 322-325.

Gondan, M., Lange, K., Rösler, F., \& Röder, B. (2004). The redundant target effect is affected by modality switch costs. Psychon Bull Rev, 11(2), 307-313.

Gondan, M., \& Minakata, K. (2016). A tutorial on testing the race model inequality. Atten Percept Psychophys, 78(3), 723-735.

Grant, K. W. (2001). The effect of speechreading on masked detection thresholds for filtered speech. The Journal of the Acoustical Society of America, 109(5), 2272-2275.

Grant, K. W., \& Seitz, P.-F. (2000). The use of visible speech cues for improving auditory detection of spoken sentences. The Journal of the Acoustical Society of America, 108(3), 1197-1208.

Grant, K. W., Walden, B. E., \& Seitz, P. F. (1998). Auditory-visual speech recognition by hearing-impaired subjects: Consonant recognition, sentence recognition, and auditory-visual integration. The Journal of the Acoustical Society of America, 103(5), 2677-2690.

Hauser, M. D., \& Marler, P. (1993). Food-associated calls in rhesus macaques (Macaca mulatta): I.

Socioecological factors. Behav. Ecol., 4(3), 194-205.

Holmes, N. P. (2009). The principle of inverse effectiveness in multisensory integration: some statistical considerations. Brain Topogr, 21(3-4), 168-176.

Janssen, P., \& Shadlen, M. N. (2005). A representation of the hazard rate of elapsed time in macaque area LIP. Nat Neurosci, 8(2), 234-241.

Luce, R. D. (1986). Response times: Their role in inferring elementary mental organization: Oxford University Press on Demand.

Ma, W. J., Beck, J. M., Latham, P. E., \& Pouget, A. (2006). Bayesian inference with probabilistic population codes. Nat Neurosci, 9(11), 1432-1438. 
Meredith, M. A., Nemitz, J. W., \& Stein, B. E. (1987). Determinants of multisensory integration in superior colliculus neurons. I. Temporal factors. J Neurosci, 7(10), 3215-3229.

Miller, J. (1982). Divided attention: Evidence for coactivation with redundant signals. Cognitive Psychology, 14(2), 247-279.

Miller, J. (1986). Timecourse of coactivation in bimodal divided attention. Percept Psychophys, 40(5), 331-343.

Miller, J. (2016). Statistical facilitation and the redundant signals effect: What are race and coactivation models? Atten Percept Psychophys, 78(2), 516-519.

Molholm, S., Ritter, W., Murray, M. M., Javitt, D. C., Schroeder, C. E., \& Foxe, J. J. (2002). Multisensory auditory-visual interactions during early sensory processing in humans: a high-density electrical mapping study. Cognitive Brain Research, 14(1), 115-128.

Murray, M. M., Molholm, S., Michel, C. M., Heslenfeld, D. J., Ritter, W., Javitt, D. C., et al. (2005). Grabbing your ear: rapid auditory-somatosensory multisensory interactions in low-level sensory cortices are not constrained by stimulus alignment. Cereb Cortex, 15(7), 963-974.

Nickerson, R. S. (1973). Intersensory facilitation of reaction time: Energy summation or preparation enhancement? Psychological Review, 80(6), 489-509.

Otto, T. U., Dassy, B., \& Mamassian, P. (2013). Principles of multisensory behavior. J Neurosci, 33(17), 74637474.

Otto, T. U., \& Mamassian, P. (2012). Noise and correlations in parallel perceptual decision making. Curr Biol, 22(15), 1391-1396.

Owen, D. B. (1980). A table of Normal integrals. Communications in Statistics - Simulation and Computation, 9, 389-419.

Populin, L. C., \& Yin, T. C. (2002). Bimodal interactions in the superior colliculus of the behaving cat. J Neurosci, 22(7), 2826-2834.

R Core Team. (2017). R: A language and environment for statistical computing. Vienna, Austria: R Foundation for Statistical Computing.

Raab, D. H. (1962). Statistical facilitation of simple reaction times. Trans N Y Acad Sci, 24, 574-590.

Raposo, D., Sheppard, J. P., Schrater, P. R., \& Churchland, A. K. (2012). Multisensory decision-making in rats and humans. J Neurosci, 32(11), 3726-3735.

Ratcliff, R. (1988). Continuous versus discrete information processing modeling accumulation of partial information. Psychol Rev, 95(2), 238-255.

Ratcliff, R., \& McKoon, G. (2008). The diffusion decision model: theory and data for two-choice decision tasks. Neural Comput, 20(4), 873-922.

Ratcliff, R., Smith, P. L., Brown, S. D., \& McKoon, G. (2016). Diffusion Decision Model: Current Issues and History. Trends Cogn Sci, 20(4), 260-281.

Ratcliff, R., Thapar, A., \& McKoon, G. (2003). A diffusion model analysis of the effects of aging on brightness discrimination. Percept Psychophys, 65(4), 523-535.

Rowell, T. E., \& Hinde, R. A. (1962). Vocal communication by the rhesus monkey (Macaca mulatta).

Proceedings of the Zoological Society London, 138, 279-294.

Sakata, S., Yamamori, T., \& Sakurai, Y. (2004). Behavioral studies of auditory-visual spatial recognition and integration in rats. Experimental Brain Research, 159(4), 409-417.

Schwartz, J.-L., Berthommier, F., \& Savariaux, C. (2004). Seeing to hear better: evidence for early audio-visual interactions in speech identification. Cognition, 93(2), B69-B78.

Schwarz, W. (1989). A new model to explain the redundant-signals effect. Percept Psychophys, 46(5), $498-500$. Schwarz, W. (1994). Diffusion, Superposition and the Redundant-Targets Effect. Journal of Mathematical Psychology, 38(4), 504-520. 
Schwarz, W. (2006). On the relationship between the redundant signals effect and temporal order judgments: parametric data and a new model. J Exp Psychol Hum Percept Perform, 32(3), 558-573.

Seilheimer, R. L., Rosenberg, A., \& Angelaki, D. E. (2014). Models and processes of multisensory cue combination. Curr Opin Neurobiol, 25, 38-46.

Shub, D. E., \& Richards, V. M. (2009). Psychophysical spectro-temporal receptive fields in an auditory task. Hear Res, 251(1-2), 1-9.

Skaliora, I., Doubell, T. P., Holmes, N. P., Nodal, F. R., \& King, A. J. (2004). Functional topography of converging visual and auditory inputs to neurons in the rat superior colliculus. J Neurophysiol, 92(5), 2933-2946.

Smith, P. L., \& Ratcliff, R. (2004). Psychology and neurobiology of simple decisions. Trends Neurosci, 27(3), 161-168.

Stanford, T. R., Quessy, S., \& Stein, B. E. (2005). Evaluating the operations underlying multisensory integration in the cat superior colliculus. Journal of Neuroscience, 25(28), 6499-6508.

Stanford, T. R., \& Stein, B. E. (2007). Superadditivity in multisensory integration: putting the computation in context. Neuroreport, 18(8), 787-792.

Stein, B. E., \& Meredith, M. A. (1993). Merging of the Senses. Cambridge, MA: MIT Press.

Stein, B. E., Meredith, M. A., Huneycutt, W. S., \& McDade, L. (1989). Behavioral Indices of Multisensory Integration: Orientation to Visual Cues is Affected by Auditory Stimuli. Journal of Cognitive Neuroscience, 1(1), 12-24.

Stein, B. E., \& Stanford, T. R. (2008). Multisensory integration: current issues from the perspective of the single neuron. Nat Rev Neurosci, 9(4), 255-266.

Townsend, J. T., \& Ashby, F. G. (1983). Stochastic modeling of elementary psychological processes: CUP Archive.

Ulrich, R., \& Miller, J. (1997). Tests of Race Models for Reaction Time in Experiments with Asynchronous Redundant Signals. J Math Psychol, 41(4), 367-381.

van Wassenhove, V., Grant, K. W., \& Poeppel, D. (2007). Temporal window of integration in auditory-visual speech perception. Neuropsychologia, 45(3), 598-607.

van Atteveldt, N., Murray, Micah M., Thut, G., \& Schroeder, Charles E. (2014). Multisensory Integration: Flexible Use of General Operations. Neuron, 81(6), 1240-1253.

Voss, A., Rothermund, K., \& Brandtstädter, J. (2008). Interpreting ambiguous stimuli: Separating perceptual and judgmental biases. Journal of Experimental Social Psychology, 44(4), 1048-1056. 